\title{
Title: Switching secretory pathway direction for organelle acquisition in plants
} 1

\section{Affiliations:}

${ }^{1}$ Division of Cellular Dynamics, National Institute for Basic Biology, Nishigonaka 38, Myodaiji,

Okazaki, Aichi, 444-8585 Japan.

${ }^{2}$ The Department of Basic Biology, SOKENDAI (The Graduate University for Advanced

Studies), Nishigonaka 38, Myodaiji, Okazaki, Aichi, 444-8585 Japan.

${ }^{3}$ Department of Biological Sciences, Graduate School of Sciences, The University of Tokyo, 7-3-

1 Hongo, Bunkyo-ku, Tokyo 113-0033, Japan.

${ }^{4}$ Department of Applied Biological Chemistry, Graduate School of Horticulture, Chiba

3 University, 648 Matsudo, Matsudo, Chiba, Japan.

${ }^{5}$ Graduate School of Biostudies, Kyoto University, Kitashirakawa-oiwake-cho, Sakyo-ku, Kyoto 606-8502, Japan.

${ }^{6}$ Functional Genomics Facility, National Institute for Basic Biology (NIBB), Okazaki, Aichi 4447858 , Japan.

${ }^{7}$ Live Cell Super-Resolution Imaging Research Team, RIKEN Center for Advanced Photonics, 9 2-1 Hirosawa, Wako, Saitama 351-0198, Japan.

*Correspondence to: tueda@nibb.ac.jp 


\section{Abstract:}

Eukaryotic cells acquired novel organelles during evolution through mechanisms that remain largely obscure. The existence of the unique oil body compartment is a synapomorphy of liverworts that represents lineage-specific acquisition of this organelle during evolution, although its origin, biogenesis, and physiological function are yet unknown. We found that two Syntaxin 1 paralogs in the liverwort, Marchantia polymorpha, are distinctly targeted to forming cell plates

\section{Main Text:}

Eukaryotic cells originated from prokaryotes by expanding their endomembrane network during evolution, with the last eukaryotic common ancestor (LECA) likely possessing a complex set of organelles ${ }^{1}$. New membrane trafficking pathways were added to the LECA endomembrane network, some of which were secondarily lost in a lineage-specific manner, resulting in divergent and organism-specific membrane trafficking systems and organelle compositions of extant eukaryotes ${ }^{2-4}$. However, it remains mostly unknown how organelles were acquired during evolution. Also in the plant lineage, several organelles and organelle functions have been uniquely acquired during evolution. For example, the plant vacuole harbours a unique function that is not shared with the animal lysosome and the yeast vacuole: storage of proteins ${ }^{5}$. This vacuolar function is fulfilled through the plant-unique vacuolar trafficking system, which 
comprises multiple vacuolar transport pathways involving plant-unique machinery components acquired during plant evolution ${ }^{6}$. The cell plate, which is formed during the mitotic phase to accomplish cytokinesis in land plants, is also a prominent example of plant-specific cellular structures/organelles ${ }^{7}$.

One of the basal-most land plant lineages, liverworts, also possess a unique organelle, the oil body, existence of which is a synapomorphy of this lineage. The liverwort oil body contains bioactive compounds such as sesquiterpenoids and cyclic bisbibenzyl compounds and is not related to the oil body that stores neutral lipids in storage organs like seeds and fruits (i.e. lipid body or oleosome). About $90 \%$ of liverwort species have this organelle; however, its origin, biogenesis, and physiological function remain unclear with controversial origins proposed from microscopic observations ${ }^{8-11}$, although the first description of the liverwort oil body dates back to $1834^{12}$. Through the systematic analysis of SNARE proteins in the liverwort, Marchantia polymorpha (hereafter referred to as Marchantia), we identified an oil body-resident protein, MpSYP12B ${ }^{13}$, which is a homolog of animal Syntaxin 1 that acts in the final step of exocytosis. Functions of SYP1 members to which MpSYP12B belongs dramatically diversified during plant evolution, suggesting expanded secretory trafficking systems in plants. Arabidopsis SYP1 consists of three subgroups, SYP11, SYP12, and SYP13. The SYP13 group mediates constitutive secretion, whereas the other groups are involved in plant-specific higher ordered functions; for example, KNOLLE (also known as SYP111) is specifically involved in membrane fusion at forming cell plates, and PEN1 (also known as SYP121) is required for intact penetration resistance against powdery mildew fungi and regulation of the ion channel activity ${ }^{14-19}$. For more insight into the functional diversification of SYP1 members in Marchantia and the 
mechanism of oil body biogenesis, we further characterized four SYP1 members in this organism 13,20.

\section{MpSYP12A is important for cell plate formation}

Our phylogenetic analysis suggested that MpSYP1 members belong to two distinct subgroups: the SYP13 group (MpSYP13A and 13B) and the SYP11/12 group (MpSYP12A and 12B) that contains SYP11 and SYP12 groups in seed plants ${ }^{21}$ (Fig. S1A). MpSYP12A, 13A and 13B were ubiquitously expressed and localized to the plasma membrane (PM) in thallus tissues, whereas MpSYP12B exhibited specific expression in a subpopulation of thallus cells (Fig S1B-E). Using some ubiquitously expressed mCitrine-MpSYP1 protein fusion constructs driven by their own regulatory elements, we detected specific localization of MpSYP12A at forming cell plates associated with the phragmoplast that rapidly stained with FM4-64 (Figs. 1A-F, S1F, and S1G). This localization suggested that MpSYP12A could be a functional counterpart of KNOLLE in Arabidopsis, which was further verified genetically.

We tried to generate a complete Mpsyp12a knockout mutant by genome editing (Fig. S1H) but were not successful, probably because it is an essential gene. However, we succeeded in isolating chimeric plants comprising mutated and wild-type cells, in which we frequently observed enlarged cells with cell wall stabs, suggesting that MpSYP12A is required for cell plate formation during cytokinesis similar to the function of KNOLLE (Fig. 1G). The KNOLLE promoter is sufficient to target non-cytokinesis-specific SYP132 to forming cell plates ${ }^{22}$. Similarly, mCitrine-tagged MpSYP13A and 13B were localized to cell plates and the PM when expressed under the MpSYP12A promoter (Fig. 1H, I). Furthermore, a similar localization was also observed when mCitrine-MpSYP13 was expressed by the MpCYCB1 promoter (Figs. 1J and 
S1I), indicating that the cell cycle-dependent transcriptional regulation is also critical for cell plate targeting of SYP1 proteins in Marchantia. We also found that clathrin light chain (CLC) tagged with mCitrine localized to the PM and trans-Golgi network in non-dividing thallus cells, as well as on forming cell plates in Marchantia as has been reported in other plants (Fig. S1J, K) 23-25. These results strongly suggested that fundamental mechanisms of cell plate formation were conserved during land plant evolution.

\section{The oil body membrane shares common properties with the plasma membrane}

Distinct from the other MpSYP1 members, MpSYP12B exhibits specific expression in oil body cells and localizes to the oil body membrane ${ }^{13}$. Oil body cells expressing a

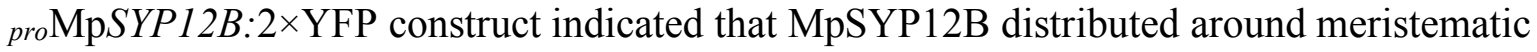
regions in young thalli, which was observed using light-sheet microscopy (Fig. 2A;

Supplementary movie S1). These results suggest that oil body formation occurs around meristematic regions and into thalli during the growth of thallus tissues. mCitrine-MpSYP12B expressed under its own regulatory elements localized to the oil body membrane with a faint signal on the PM (Fig. 2B), which was also confirmed by immuno-EM analysis using an antiGFP antibody (Fig. 2C, D). We then tested whether organelle markers for the endoplasmic reticulum (MpSEC20, MpUSE1A, MpSEC22, mRFP-HDEL, and GFP-HDEL), Golgi apparatus (MpGOS11 and MpSFT1), trans-Golgi network (MpSYP6A and MpSYP4), and tonoplast (MpSYP2 and MpVAMP71) were targeted to the oil body membrane, none of which were detected (Figs. 2E and S2B, C). Using transmission electron microscopy, we found that clathrincoated vesicles formed from the oil body membrane, and emergence and disappearance of 
112 clathrin-positive foci at the oil body membrane was also observed in transgenic plants expressing

113 Citrine-tagged MpCLC (Fig. 2G; Supplementary movie S2).

114 Given that clathrin-mediated endocytosis occurs at the PM and SYP1 members generally

115 function on the PM, the oil body membrane could share similar characteristics with the PM. This

116 hypothesis was supported by dual localization of the PM proteins, PM-type aquaporin MpPIP2

117 (Fig. 2H) and PM-resident SNARE MpSYP13A (Fig. 3A) driven by their own regulatory

118 elements. The plasma membrane-like nature of the oil body membrane and the existence of the

119 SYP1 member that is homologous to KNOLLE suggested that the oil body could be formed by

120 the fusion of secretory vesicles similar to the cell plate. Thus, the luminal space of the oil body

121 should be topologically equivalent to the extracellular space, which was tested by expressing a

122 general secretion marker sec-mRFP, which is composed of the signal peptide for ER

123 translocation and mRFP under the constitutive MpEF1 $\alpha$ promoter. In non-oil body cells, mRFP

124 fluorescence was detected only in the extracellular space (Fig. S2D). However, mRFP

125 accumulated in the oil body in addition to the extracellular space in oil body cells, demonstrating

126 equivalent topology between the lumen of the oil body and extracellular space (Fig. 2I). Unlike

127 MpSYP12A and KNOLLE, MpSYP12B loss of function did not result in a detectable

128 abnormality in oil body formation and transport of MpSYP13A or sec-mRFP (Fig. S3), probably

129 reflecting a functional redundancy between MpSYP12B and 13A similar to the partial functional 130 redundancy of KNOLLE and SYP132 ${ }^{26}$.

132 The oil body is formed by directional switching of the secretory pathway

133 Distinct from MpSYP13A with the dual localization to the plasma and oil body membranes, the 134 close homolog MpSYP13B was only localized to the PM in oil body cells (Fig. 3A, B). 
Surprisingly, both of these proteins were targeted predominantly to the oil body membrane when expressed under the regulation of the MpSYP12B promoter (Fig. 3C, D). This effect was not restricted to SYP1 homologs; the PM-resident protein MpPIP2 and a general secretion cargo secmRFP were also targeted almost exclusively to the oil body when driven by the MpSYP12B promoter (Fig. 3E, F). These results indicated that the secretory pathway is redirected inward and vesicles fuse with each other to form the oil body during the phase in which the MpSYP12B promoter is active. In contrast, when $\mathrm{Mp} S Y P 13 B$ promoter is active, the secretory pathway is directed to the PM and extracellular space, which was further confirmed by accumulation of secmRFP predominantly in the extracellular space when the construct was driven by the MpSYP $13 B$ promoter (Fig. 3G). Other organelle markers did not change their localization even when expressed under the MpSYP12B promoter (Fig. S2B, C). These results indicated that the switching of directions of the secretory pathway in oil body cells is regulated at the transcription level. The switching of secretory directions should take place periodically and repeatedly through oil body cell development, because we never observed oil body membrane localization for MpSYP13B at any developmental stages of oil body cells (Fig. 4A-C), and increase in the size of the oil body cell along with the increase in the oil body size was observed (Fig. 4D). Based on these findings, we propose the "oil body cycle hypothesis", which states that Marchantia oil body cells cycle between two distinct cellular phases: the "PM phase" in which the secretory pathway is oriented to the PM and extracellular space, and the "oil body phase" when the secretory pathway is oriented to the oil body, with the phase transition under the regulation of a transcriptional regulatory system (Fig. 4E, F). 


\section{MpERF13 transcription factor regulates oil body formation}

To investigate the regulatory system of oil body formation and oil body cycle, we screened mutants defective in oil body formation (Fig. S4A). From 48,825 T-DNA insertion lines, we identified a mutant (Mperf13 $3^{G O F}$ ) with an increased number of oil bodies. The mutant gemmae contained $334.4 \pm 85.6$ oil bodies (mean \pm SD), whereas wild-type gemmae possessed $51.9 \pm 8.7$ oil bodies under our experimental conditions (Fig. 5A-E). The T-DNA was inserted 2674-bp upstream of the start codon of Mapoly0060s0052.1 (MpERF13), which encodes a putative ERF/AP2 transcription factor in the subgroup containing DREB1A and TINY in Arabidopsis (Fig. S4B). Moreover, MpERF13 and MpSYP12B transcripts accumulated in this mutant compared to wild type as detected by RNA-sequencing (RNA-Seq) and quantitative RT-PCR analyses (Fig. S5B). We then generated knockout mutants in which the MpERF13 gene was deleted by genome editing (Fig. S4C). Two independent mutants (Mperf13-1 $1^{g e}$ and Mperf13-2 ${ }^{g e}$ ) exhibited no detectable abnormalities in thallus development and reproductive growth; however, these mutants completely lacked oil bodies in the gemma and thallus tissues (Figs. 5C-E and S4F, G). These phenotypes of gain- and loss-of-function mutations suggested that MpERF13 is a major transcription factor regulating oil body formation. Consistently, the $\mathrm{Mp} E R F 13$ promoter was highly active in oil body cells (Fig. 5F).

As MpERF13 is homologous to ERF/AP2 transcription factors, this protein was expected to be involved in the transcriptional regulation of oil body formation and/or the oil body cycle. To identify genes downstream of MpERF13, we analyzed transcriptomes in Tak-1 (wild type) and Mperf1 $3^{G O F}$ and Mperf13-1 $1^{g e}$ mutant lines by RNA-Seq. Through the comparison between the three groups using an ANOVA-like test, we identified 136 differentially-expressed genes (DEGs) other than MpERF13 whose expression was higher than two $\log _{2}$-fold change (FC) in 

Mperf13 ${ }^{G O F}$ compared with Tak-1, and lower than $-2 \log _{2} \mathrm{FC}$ in Mperf13-1 ${ }^{\text {ge }}$ than Tak-1 (FDR < 0.01) (Supplementary Table S1). To verify the RNA-Seq result, we also performed the quantitative RT-PCR analysis for selected 11 DEGs including MpERF13 and MpSYP12B, which confirmed that all of these DEGs were highly expressed in Mperf1 $3^{G O F}$, but their expression was 
constituents. We fed starved Armadillidium vulgare (pill bug) with wild-type liverwort and genetically established mutants that contain extra or no oil bodies. Thalli of Tak-1 and Mperf13 $3^{G O F}$ remained almost intact after $24 \mathrm{~h}$ of herbivory, while the areas of Mperf13-1 $1^{\text {ge }}$ and Mperf13-2 ge thalli were significantly reduced after herbivory (Figs. 6 and S6). This result demonstrated that the oil body is effective in protecting liverworts from herbivores.

We demonstrate that plants have acquired at least two organelles, the cell plate and oil body, through a common evolutionary pathway of redirecting secretory patterns from an outward to an inward direction in cells. The functions of these organelles are totally different but they share several important traits that include specific SYP11/12 members that reside at their membranes, clathrin-coated vesicles generated from the plasma and internal membranes, and both the cell plate and oil body are formed by reorientation of secretory pathways. In both cases, orientation of the secretory pathway is switched in response to transitioning cellular states, during the cell cycle for cell plate formation and the oil body cycle for oil body formation. Orientation of the plant secretory pathway is also modulated during plant-microbe interactions; symbiotic arbuscular mycorrhizal fungi redirects the plant secretory pathway to form the arbuscule in host plant cells that acts as an interface for sugar and mineral exchange ${ }^{31,32}$. Thus, redirection of the secretory pathway is a common strategy for plants to acquire novel organelles/cellular structures that had resulted in maximizing fitness for land plants during evolution. The liverwort oil body is a nice system to evaluate how novel organelles have been acquired during evolution, and the knowledge obtained from this study is useful for engineering new organelles/cellular structures in plants to maximize plant cellular functions and provides a powerful methodology for future organelle technology. 
Acknowledgments: We thank Dr. Ikuko Hara-Nishimura (Konan University), Dr. Kimitsune Ishizaki (Kobe University), Dr. Ryuichi Nishihama (Kyoto University), and Dr. Shigeo S. Sugano for sharing vectors and plant materials, Ms. Miwako Matsumoto, Ms. Asaka Akita, and Functional Genomics Facility (NIBB) for technical assistance in RNA-Seq experiments, and Dr. Ken Naito (National Agriculture and Food Research Organization) for instruction for RNA-Seq data analysis. We thank Dr. John L. Bowman (Monash University) and Dr. Facundo Romani (Universidad Nacional del Litoral) for helpful discussion. We also thank Dr. Teruyuki Niimi (NIBB) and Dr. Takahisa Miyatake (Okayama University) for identification of the species of pill bug used in this study. The support of plant cultivation rooms was provided by the Model Plant Research Facility of NIBB.

Author contributions: T.Ka. performed the majority of the experiments, H.M. performed the

Funding: This work was financially supported by Grants-in-Aid for Scientific Research from the Ministry of Education, Culture, Sports, Science, and Technology of Japan (to T.U., 19H05670, 19H05675, and 18H02470, and T.Ka., 18K14738). experiment presented in Fig. 1A-G, K.E. and T.L.S. provided essential ideas for experiments using sec-mRFP targeting and oil body detection, respectively, S.I. generated the Mpsyp $12 b-1$ mutant, K.Y. and S.S. designed the RNA-seq experiment, N.M. examined the effect of Mperf13 mutations on reproductive growth, T.Ka. and T.U. wrote the manuscript, and T.Ko., A.N., and T.U. supervised the study. 
bioRxiv preprint doi: https://doi.org/10.1101/2020.03.02.956961; this version posted May 26, 2020. The copyright holder for this preprint (which was not certified by peer review) is the author/funder. All rights reserved. No reuse allowed without permission.

249 Competing interests: Authors declare no competing interests.

251 Data and materials availability: All reads of RNA-Seq are available through the Sequence

252 Read Archive (SRA) under the accession number DRA009193. 


\section{References:}

2551 Koumandou, V. L. et al. Molecular paleontology and complexity in the last eukaryotic common ancestor. Critical reviews in biochemistry and molecular biology 48, 373-396, doi:10.3109/10409238.2013.821444 (2013).

2 Kanazawa, T. \& Ueda, T. Exocytic trafficking pathways in plants: why and how they are redirected. The New phytologist 215, 952-957, doi:10.1111/nph.14613 (2017).

L'Hernault, S. W. Spermatogenesis. WormBook: the online review of C. elegans biology, 1-14, doi:10.1895/wormbook.1.85.1 (2006).

5 Shimada, T., Takagi, J., Ichino, T., Shirakawa, M. \& Hara-Nishimura, I. Plant Vacuoles. Annual review of plant biology 69, 123-145, doi:10.1146/annurev-arplant-042817040508 (2018).

6 Minamino, N. \& Ueda, T. RAB GTPases and their effectors in plant endosomal transport. Current opinion in plant biology 52, 61-68, doi:10.1016/j.pbi.2019.07.007 (2019). bryophytes. Phytochemistry 91, 52-80, doi:10.1016/j.phytochem.2012.04.012 (2013). 
9 He, X. L., Sun, Y. \& Zhu, R. L. The Oil Bodies of Liverworts: Unique and Important Organelles in Land Plants. Critical Reviews in Plant Sciences 32, 293-302, doi:10.1080/07352689.2013.765765 (2013).

10 Suire, C. A comparative, transmission-electron microscopic study on the formation of oilbodies in liverworts. J Hattori Bot Lab, 209-232 (2000).

11 Tanaka, M. et al. Direct evidence of specific localization of sesquiterpenes and marchantin A in oil body cells of Marchantia polymorpha L. Phytochemistry 130, 77-84, doi:10.1016/j.phytochem.2016.06.008 (2016).

12 Hübener, J. W. P. Hepaticologia germanica, oder Beschreibung der Deutschen lebermoose. Schwan \& Götz'sche Hofbuchlandlung, Mannheim (1834).

13 Kanazawa, T. et al. SNARE Molecules in Marchantia polymorpha: Unique and Conserved Features of the Membrane Fusion Machinery. Plant \& cell physiology 57, 307-324, doi:10.1093/pcp/pcv076 (2016).

14 Assaad, F. F. et al. The PEN1 syntaxin defines a novel cellular compartment upon fungal attack and is required for the timely assembly of papillae. Mol Biol Cell 15, 5118-5129, doi:10.1091/mbc.E04-02-0140 (2004).

15 Collins, N. C. et al. SNARE-protein-mediated disease resistance at the plant cell wall. Nature 425, 973-977, doi:10.1038/nature02076 (2003).

16 Enami, K. et al. Differential expression control and polarized distribution of plasma membrane-resident SYP1 SNAREs in Arabidopsis thaliana. Plant \& cell physiology 50, 280-289, doi:10.1093/pcp/pcn197 (2009). 
17 Grefen, C. et al. A vesicle-trafficking protein commandeers Kv channel voltage sensors for voltage-dependent secretion. Nature plants 1, 15108, doi:10.1038/nplants.2015.108 (2015).

18 Lauber, M. H. et al. The Arabidopsis KNOLLE protein is a cytokinesis-specific syntaxin. Journal of Cell Biology 139, 1485-1493, doi:DOI 10.1083/jcb.139.6.1485 (1997).

19 Lukowitz, W., Mayer, U. \& Jurgens, G. Cytokinesis in the Arabidopsis embryo involves the syntaxin-related KNOLLE gene product. Cell 84, 61-71, doi:Doi 10.1016/S00928674(00)80993-9 (1996).

20 Bowman, J. L. et al. Insights into Land Plant Evolution Garnered from the Marchantia polymorpha Genome. Cell 171, 287-304 e215, doi:10.1016/j.cell.2017.09.030 (2017).

21 Slane, D., Reichardt, I., El Kasmi, F., Bayer, M. \& Jurgens, G. Evolutionarily diverse SYP1 Qa-SNAREs jointly sustain pollen tube growth in Arabidopsis. The Plant journal : for cell and molecular biology 92, 375-385, doi:10.1111/tpj.13659 (2017).

22 Reichardt, I. et al. Mechanisms of Functional Specificity Among Plasma-Membrane Syntaxins in Arabidopsis. Traffic 12, 1269-1280, doi:10.1111/j.1600-0854.2011.01222.x (2011).

23 Ito, E. et al. Dynamic behavior of clathrin in Arabidopsis thaliana unveiled by live imaging. The Plant journal : for cell and molecular biology 69, 204-216, doi:10.1111/j.1365-313X.2011.04782.x (2012). Three-dimensional analysis of syncytial-type cell plates during endosperm cellularization 
visualized by high resolution electron tomography. The Plant cell 13, 2033-2051, doi:10.1105/tpc.010150 (2001).

25 Samuels, A. L., Giddings, T. H., Jr. \& Staehelin, L. A. Cytokinesis in tobacco BY-2 and root tip cells: a new model of cell plate formation in higher plants. The Journal of cell biology 130, 1345-1357, doi:10.1083/jcb.130.6.1345 (1995).

26 Park, M. et al. Concerted Action of Evolutionarily Ancient and Novel SNARE Complexes in Flowering-Plant Cytokinesis. Developmental cell 44, 500-511 e504, doi:10.1016/j.devcel.2017.12.027 (2018).

27 Adebesin, F. et al. Emission of volatile organic compounds from petunia flowers is (2017).

28 Kang, J. et al. Plant ABC Transporters. The arabidopsis book 9, e0153, doi:10.1199/tab.0153 (2011).

29 Kubo, H. et al. Biosynthesis of riccionidins and marchantins is regulated by R2R3-MYB transcription factors in Marchantia polymorpha. Journal of plant research 131, 849-864, doi:10.1007/s10265-018-1044-7 (2018).

30 Stahl, E. Pflanzen und Schnecken. Jena. Z. Med. Naturwiss 22, 557 - 684 (1888).

31 Ivanov, S. \& Harrison, M. J. A set of fluorescent protein-based markers expressed from constitutive and arbuscular mycorrhiza-inducible promoters to label organelles, membranes and cytoskeletal elements in Medicago truncatula. The Plant journal : for cell and molecular biology 80, 1151-1163, doi:10.1111/tpj.12706 (2014). 
338 Pumplin, N., Zhang, X., Noar, R. D. \& Harrison, M. J. Polar localization of a symbiosisspecific phosphate transporter is mediated by a transient reorientation of secretion. Proceedings of the National Academy of Sciences of the United States of America 109, E665-672, doi:10.1073/pnas.1110215109 (2012). 


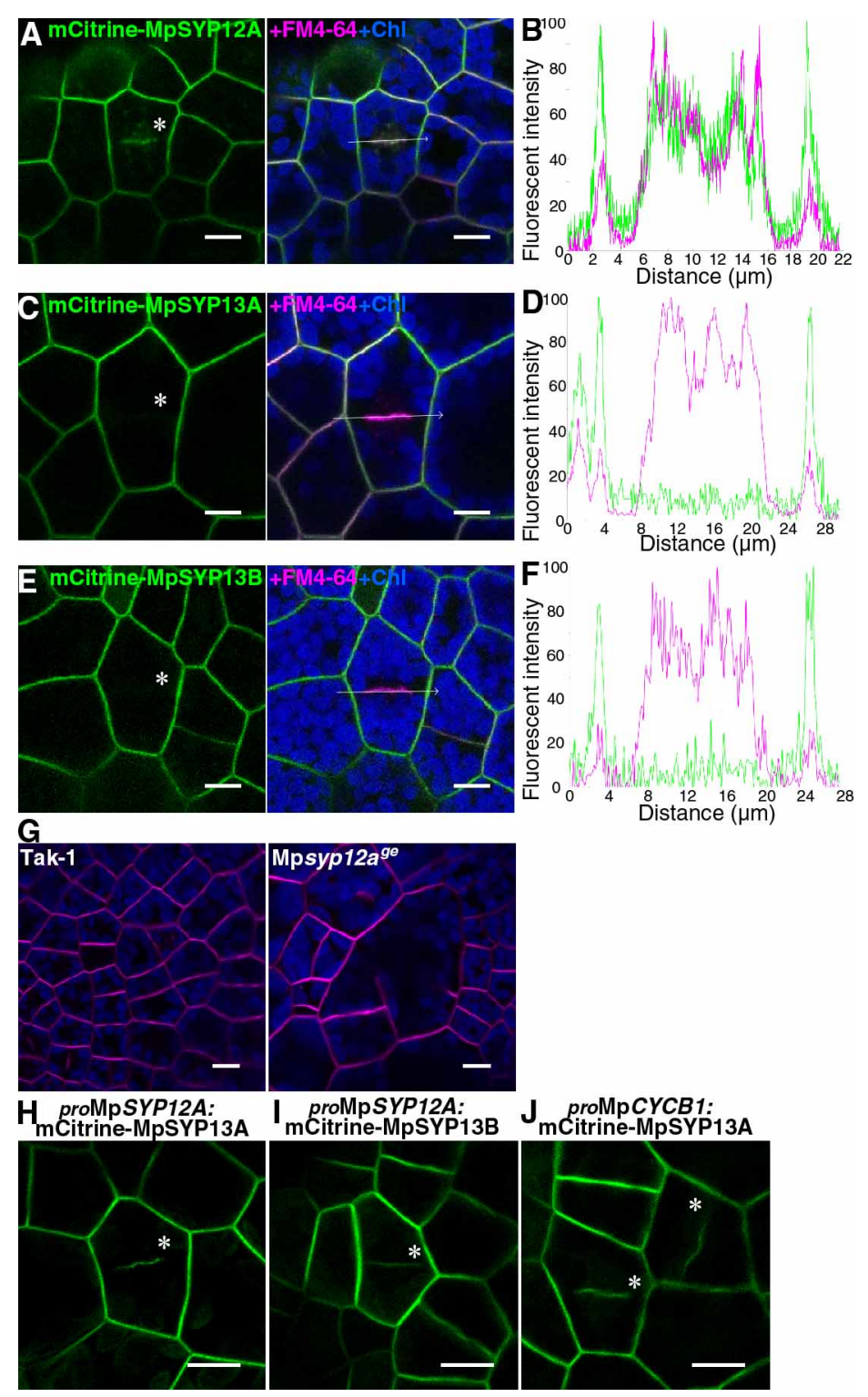

Fig. 1. MpSYP12A is important for cell plate formation in M. polymorpha thallus cells. (A,

relative fluorescence intensities from mCitrine and FM4-64 along the white arrows in panels A (B), C (D), and E (F). (G) Thallus cells of Tak-1 (left) and a putative knock-out mutant (right) stained with FM4-64. (H and I) Dividing thallus cells expressing mCitrine-MpSYP13A (H) or mCitrine-MpSYP13B (I) driven by the MpSYP12A promoter. (J) Dividing thallus cells 
350 expressing mCitrine-MpSYP13A under the regulation of the MpCYCB1 promoter. Asterisks

351 indicate forming cell plates. Green, magenta, and blue pseudo-colors indicate fluorescence from

352 mCitrine, FM4-64, and chlorophyll, respectively. Bars $=10 \mu \mathrm{m}$. 


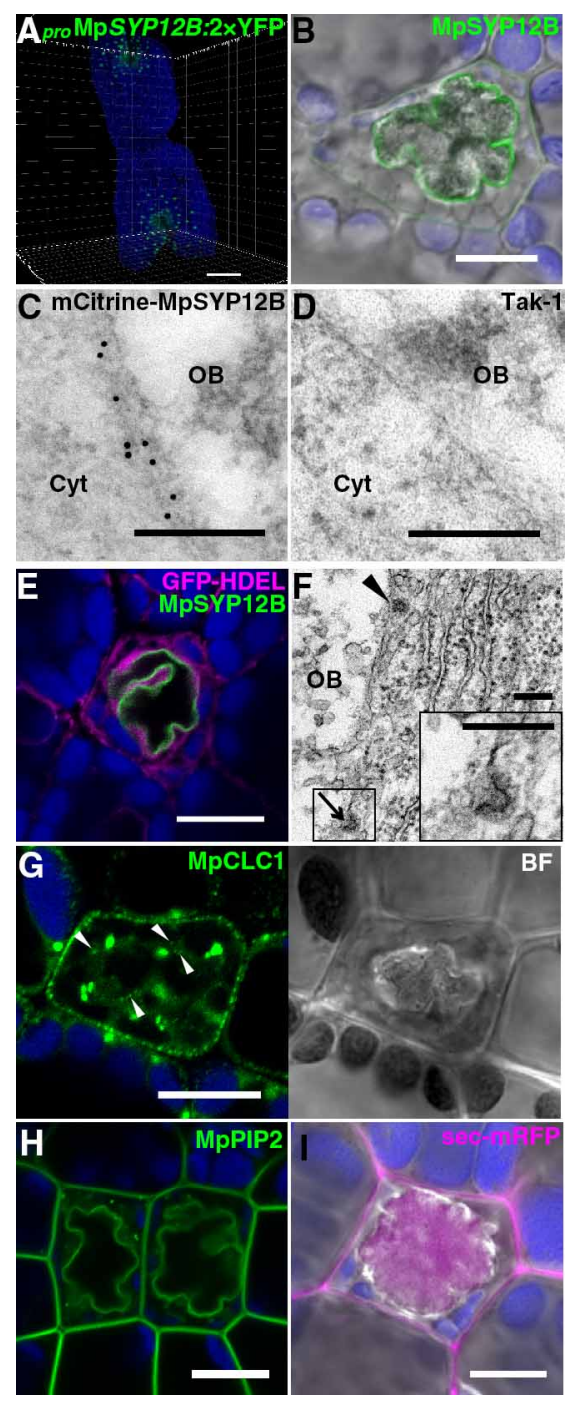
MpSYP12B under its own regulatory elements. The bright field image was merged. (C and D) Immunoelectron micrographs of oil body cells in thalli of Tak-1 with (C) or without (D) mCitrine-MpSYP12B expression. Cyt, cytosol, and OB, oil body. (E) An oil body cell expressing GFP-HDEL (magenta) and mCitrine-MpSYP12B (green). (F) An electron micrograph of the oil body cell. The arrowhead and arrow indicate a clathrin-coated vesicle and a clathrin-coated pit, respectively. The magnified image of the boxed region is shown in the inset. 
363 (G) An oil body cell expressing MpCLC1-Citrine. Arrowheads indicate clathrin-coated $364 \quad$ vesicles/pits on the oil body membrane. BF, bright field image. (H) Oil body cells expressing 365 mCitrine-MpPIP2 under its own regulatory elements. (I) An oil body cell expressing sec-mRFP 366 driven by the $\mathrm{MpEF1} \alpha$ promoter. The bright field image was merged. The blue pseudo-color 367 indicates chlorophyll autofluorescence. Bars $=500 \mu \mathrm{m}$ in $(\mathrm{A}), 10 \mu \mathrm{m}$ in $(\mathrm{B}),(\mathrm{E})$, and (G - I), and $368200 \mathrm{~nm}$ in (C), (D), and (F). 

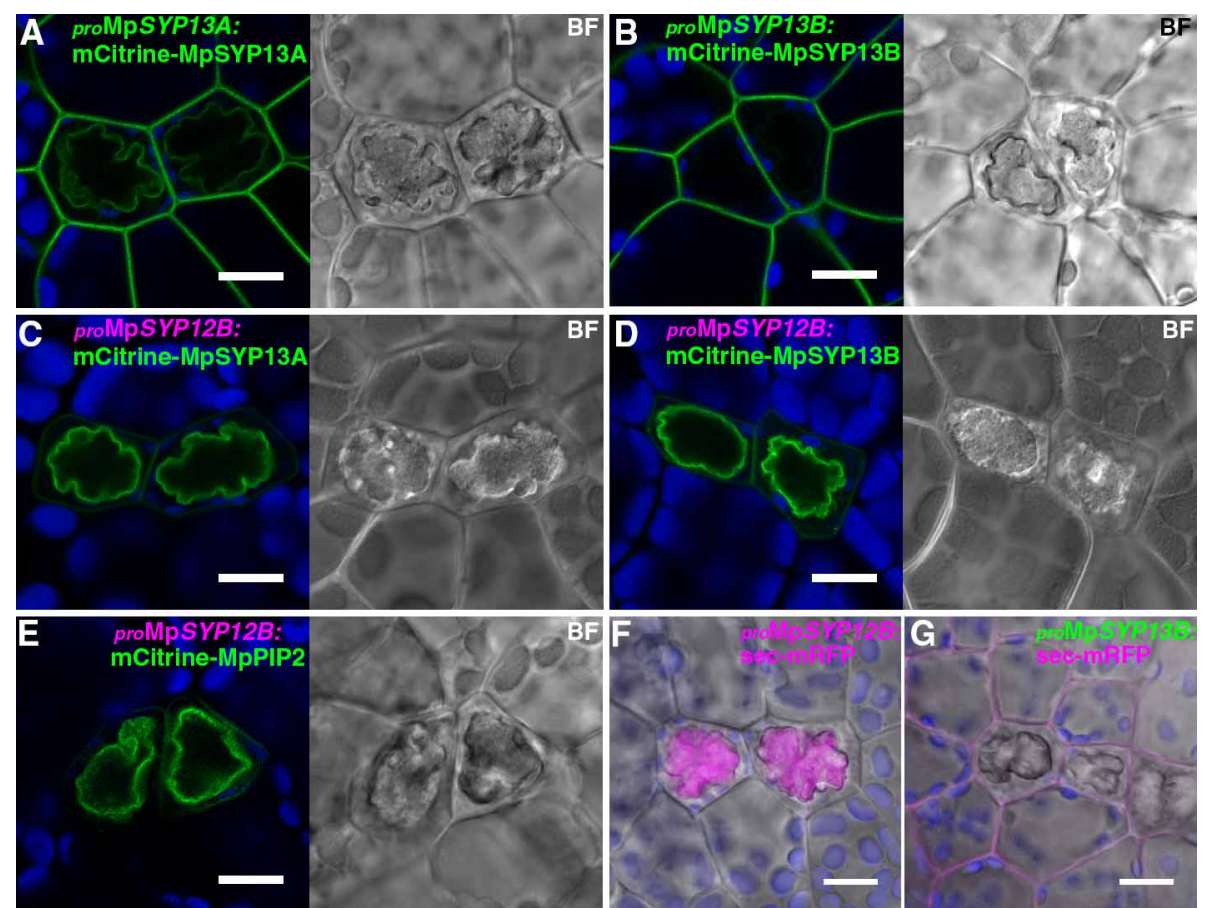

Fig. 3. The oil body is formed by directional switching of the secretory pathway (A and B)

MpSYP13B (B) under their own regulatory elements. (C - E) Thallus cells including oil body 

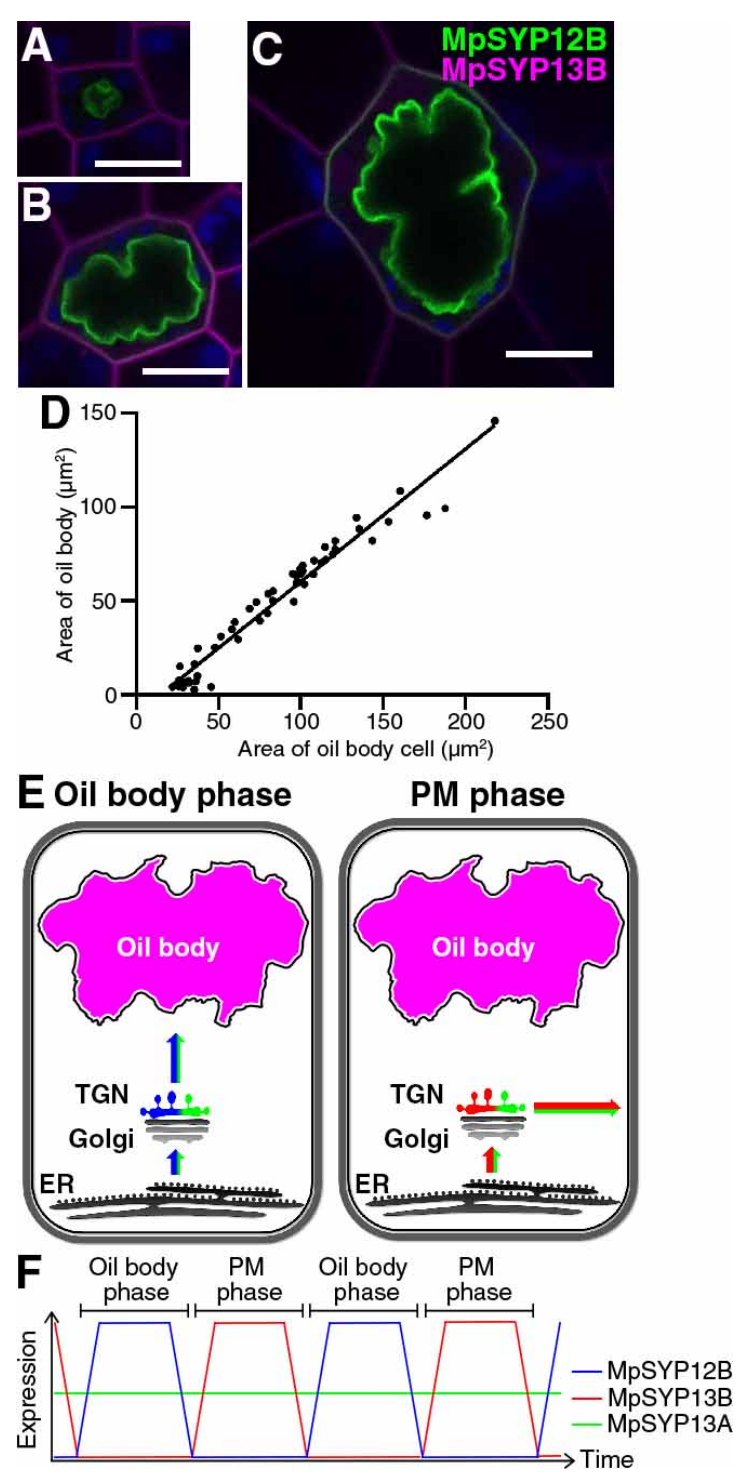

Fig. 4. The oil body cycle hypothesis (A - C) Oil body cells at distinct developmental stages expressing mCitrine-MpSYP12B and mRFP-MpSYP13B. Green, magenta, and blue pseudocolors indicate mCitrine, mRFP, and autofluorescence from chlorophyll, respectively. Bars $=10$ $\mu \mathrm{m}$. (D) A scatter diagram of the areas of oil body cells on the $X$ axis and the areas of oil bodies on the $\mathrm{Y}$ axis. $\mathrm{R}^{2}=0.9547$. $(\mathrm{E}$ and $\mathrm{F}$ ) Schemes of the oil body cycle hypothesis. Newly synthesized PM proteins and secreted cargos are targeted to the oil body membrane in the oil body phase, and then to the PM and extracellular space during the PM phase (E). Expression levels of MpSYP12B and MpSYP13B periodically oscillate depending on the cell phases; 
bioRxiv preprint doi: https://doi.org/10.1101/2020.03.02.956961; this version posted May 26, 2020. The copyright holder for this preprint (which was not certified by peer review) is the author/funder. All rights reserved. No reuse allowed without permission.

387 whereas, MpSYP13A is constitutively expressed, resulting in dual localization at the oil body 388 membrane and the PM (F). 


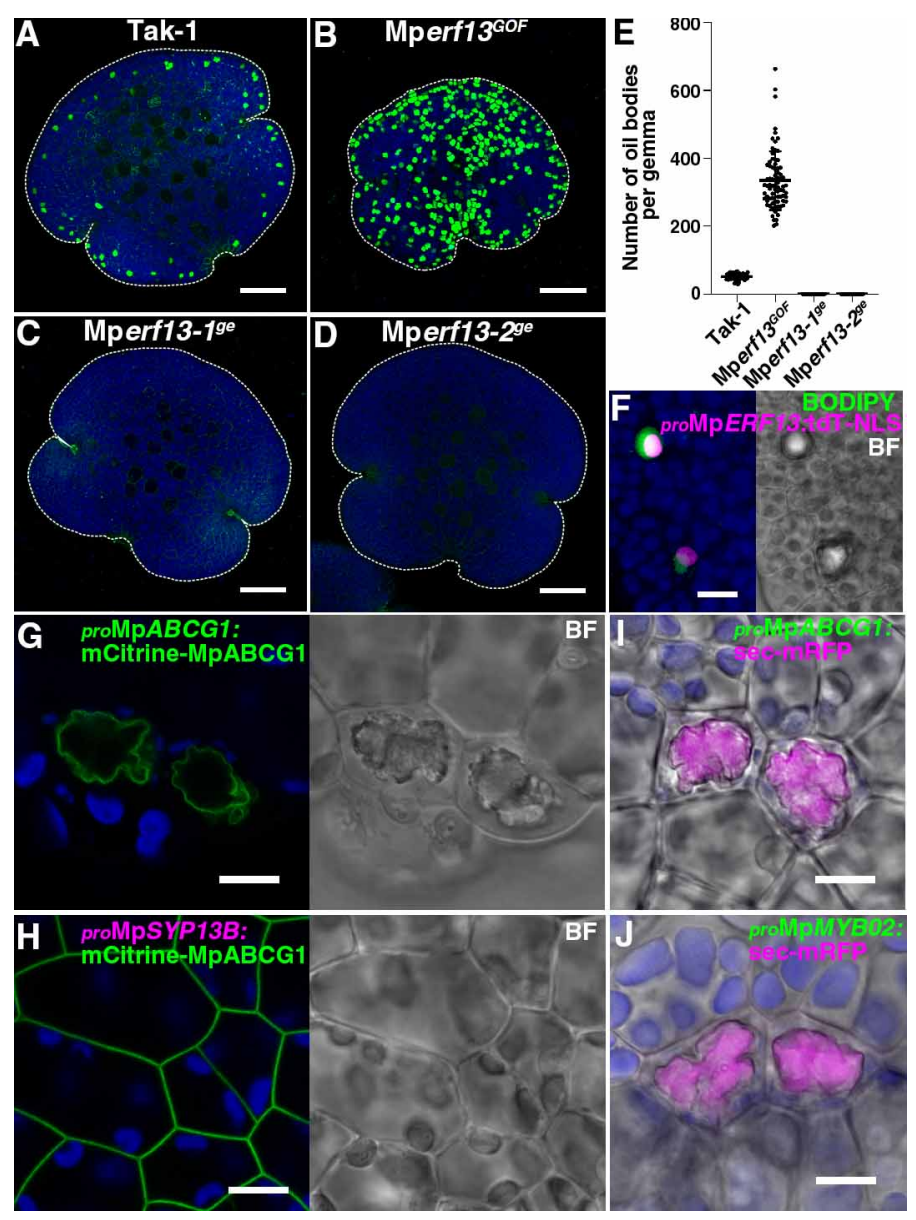

Fig. 5. MpERF13 transcription factor regulates oil body formation. (A - D) Maximum intensity projection images of BODIPY-stained gemmae of Tak-1 (A), Mperf13 ${ }^{G O F}$ (B), staining in gemmae. Bars indicate means \pm SD. Statistical analyses between Tak-1 and each $1.19 \times 10^{-43}$ for Mperf13 ${ }^{G O F}, 1.44 \times 10^{-45}$ for Mperf13-1 $1^{g e}$, and $1.44 \times 10^{-45}$ for Mperf13-2 $2^{g e}$. (F) A maximum intensity projection image of oil body and non-oil body cells in a gemma expressing genotype were conducted using a two-tailed Welch's $t$-test. Sample numbers were 57 gemmae for Tak-1, 78 for Mperf13 $3^{G O F}, 61$ for Mperf13-1 ${ }^{g e}$, and 75 for Mperf13-2 ${ }^{\text {ge }}$; and $p$-values are tandemTomato (tdT)-NLS driven by the MpERF13 promoter. ( $\mathrm{G}$ and $\mathrm{H}$ ) Thallus cells including oil body cells expressing mCitrine-MpABCG1 under its own regulatory elements $(\mathrm{G})$ and the MpSYP13B promoter $(\mathrm{H})$. BF, bright field images. (I and J) Thallus cells expressing sec-mRFP 
401 under the MpABCG1 (I) and the MpMYBO2 (J) promoters. Bright field images are merged.

402 Green, magenta, and blue pseudo-colors indicate fluorescence from BODIPY or mCitrine, tdT or

403 mRFP, and chlorophyll, respectively. Bars $=100 \mu \mathrm{m}$ in $(\mathrm{A}-\mathrm{D})$ and $10 \mu \mathrm{m}$ in $(\mathrm{F}-\mathrm{J})$.

404 

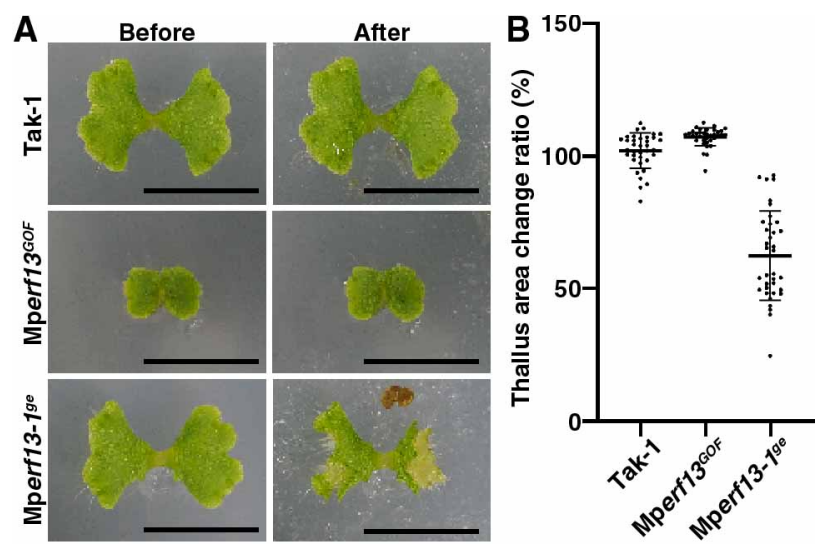

Fig. 6 (A) Ten-day-old thalli in indicated genotypes (Before) they were fed to starved pill bugs or $24 \mathrm{~h}$ after feeding (After). (B) Quantification of fluorescence signals images in panel (A). Ratios of thallus areas of "After" to "Before" pill bug feeding were calculated to indicate thallus area change ratios $(n=36$ thalli for each genotype). Bars indicate means \pm SD. Statistical analyses between Tak-1 and each genotype were conducted by two-tailed Welch's $t$-test. The $p$ values were $5.21 \times 10^{-4}$ for Mperf13 $3^{G O F}$ and $1.05 \times 10^{-16}$ for Mperf13-1 ${ }^{g e}$. Bars $=1 \mathrm{~cm}$. 


\section{Supplementary Information for}

Switching secretory pathway direction for organelle acquisition in plants

Takehiko Kanazawa, Hatsune Morinaka, Kazuo Ebine, Takashi L. Shimada, Sakiko Ishida, Naoki Minamino, Katsushi Yamaguchi, Shuji Shigenobu, Takayuki Kohchi, Akihiko Nakano, Takashi Ueda.

Correspondence to: tueda@nibb.ac.jp

\section{This PDF file includes:}

Materials and Methods

Supplementary Text

Figs. S1 to S6

Captions for Tables S1 \& S2

Captions for Movies S1 \& S2

References 33-54

\section{Other Supplementary Materials for this manuscript include the following:}

Movies S1 \& S2

Table S1 \& S2 


\section{Materials and Methods}

Phylogenetic Analyses

Phylogenetic analyses were performed as previously described in Bowman et al, $2017^{20}$ with minor modification. Previously published datasets in Kanazawa et al., 2016 and Bowman et al., 2017, were used for phylogenetic analyses of SYP1 and ERF/AP2 members, respectively ${ }^{13,20}$. Multiple sequence alignments were performed using the MUSCLE program version 3.8.31 33,34 with the default parameter. The alignment gaps were removed by Gblocks program version $0.91 b^{35,36}$ or manually. The maximum likelihood phylogenetic analyses were performed using PhyML $3.0{ }^{37}$ under the LG model. Bootstrap analyses were performed by resampling 1000 sets.

\section{$\underline{\text { Vector Construction }}$}

Open reading frames (ORFs) and genomic sequences of Marchantia genes were amplified by polymerase chain reaction (PCR) from cDNA and genomic DNA prepared from the accession Tak-1, and the amplified products were subcloned into pENTR/D-TOPO (ThermoFisher, Waltham, USA) according to the manufacturer's instructions. The primer sequences and sizes of amplified products are listed in Supplementary Table S2. For construction of pENTR

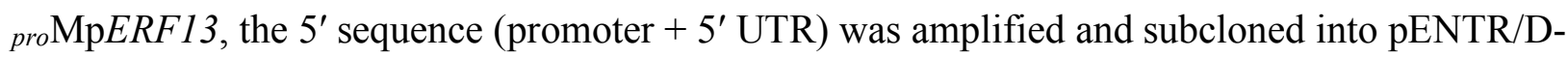
TOPO. The resultant sequence was introduced into pMpGWB316 ${ }^{38}$ using the Gateway LR Clonase $^{\mathrm{TM}}$ II Enzyme Mix (ThermoFisher) according to the manufacturer's instructions. For construction of pENTR genomic mGFP-MpSYP12A and mRFP-MpSYP13B constructs, the cDNA for mGFP and mRFP were inserted into SmaI and BamHI sites in the pENTR genomic XFP(SmaI)-MpSYP12A and pENTR genomic XFP(BamHI)-MpSYP13B, which were previously prepared ${ }^{13}$ using the In-Fusion HD Cloning System (Clontech, Shiga, Japan). For construction of pENTR genomic mCitrine-MpPIP2, pENTR genomic mCitrine-MpSYP4, pENTR genomic mCitrine-MpSEC22, and pENTR genomic mCitrine-MpABCG1, genomic sequences of the protein-coding regions and 3' flanking sequences were amplified with a SmaI site at the $5^{\prime}$ end and subcloned into the pENTR vector. The 5' sequences (promoter $+5^{\prime}$ UTR) and cDNA of mCitrine were amplified and inserted into Not I and SmaI sites, respectively, of the resultant pENTR vectors using the In-Fusion HD Cloning System. The resultant chimeric genes were then introduced into pMpGWB301 ${ }^{38}$ using the Gateway LR Clonase ${ }^{\mathrm{TM}}$ II Enzyme Mix. For construction of $\mathrm{pENTR}$ pro MpSYP12B:mCitrine, the pro MpSYP12B:mCitrine sequence was 
amplified from the $\mathrm{pENTR}$ genomic mCitrine-MpSYP12B vector, and subcloned into pENTR/D-TOPO. The resultant sequence was introduced into pMpGWB307 ${ }^{38}$ using the Gateway LR Clonase ${ }^{\mathrm{TM}}$ II Enzyme Mix to create the pMpGWB307 proMpSYP12B:2×YFP construct. For construction of pENTR genomic MpCLC1-mCitrine, genomic sequence comprising the $5^{\prime}$ flanking (promoter $+5^{\prime}$ UTR) and protein-coding sequences were amplified with a SmaI site at the $3^{\prime}$ end and subcloned into the pENTR vector. The $3^{\prime}$ flanking sequence including 3' UTR and cDNA for mCitrine were amplified and inserted into AscI and SmaI sites, respectively, of the resultant pENTR vectors using the In-Fusion HD Cloning System. The resultant chimeric genes were then introduced into pMpGWB301 ${ }^{38}$ using the Gateway LR Clonase $^{\mathrm{TM}}$ II Enzyme Mix. For construction of pENTR sec-mRFP, sequences for the signal peptide and mRFP were independently amplified by PCR, and the sequence of sec-mRFP was then amplified by PCR using the mixture of the amplified products as templates. The amplified products were subcloned into pENTR/D-TOPO. For the construction of pENTR mRFP-HDEL, the sequence of SP-mRFP-HDEL was amplified by PCR using pENTR sec-mRFP as template, and subcloned into $\mathrm{pENTR/D-TOPO}$. For construction of pENTR proMpSYP12B:sec-mRFP, pENTR ${ }_{p r o} \mathrm{Mp} S Y P 13 B$ :sec-mRFP, pENTR ${ }_{p r o} \mathrm{Mp} A B C G 1$ :sec-mRFP, and pENTR pro MpMYB02:sec-mRFP, the 5' sequences (promoter + 5' UTR) were amplified and were inserted into NotI sites of the pENTR sec-mRFP vector using the In-Fusion HD Cloning System. The resultant sequences were then introduced into pMpGWB301 ${ }^{38}$ using the Gateway LR Clonase $^{\mathrm{TM}}$ II Enzyme Mix. To construct pMpGWB301-derived Gateway vectors (Fig. S2A), the promoter:mCitrine sequences were amplified from genomic constructs described above, and inserted at a HindIII site of pMpGWB301 using the In-Fusion HD Cloning System. pENTR MpTUB2 was kindly provided from Dr. R. Nishihama and introduced into pMpGWB301 pro $_{\text {MpSYP2:mCitrine-GW (Fig. S2A) using the Gateway LR Clonase }}{ }^{\mathrm{TM}}$ II Enzyme Mix. For construction of the genome-editing vectors, the target sequences were selected using CRISPR direct (https://crispr.dbcls.jp/) ${ }^{39}$, and double-stranded oligonucleotides of the target sequences were inserted into the pMpGE_En03 vector ${ }^{40}$. The resultant gRNA cassettes were introduced into the pMpGE010 or pMpGE011 vectors ${ }^{40}$ using the Gateway LR Clonase II Enzyme Mix. For construction of the homologous recombination-mediated gene targeting vector, the 3.5-kb homologous genomic sequences were amplified from the Tak-1 genome and inserted at $P a c \mathrm{I}$ and $\mathrm{AscI}$ sites of the pJHY-TMp1 vector ${ }^{41}$ using the In-Fusion HD Cloning System. 


\section{$\underline{\text { Plant materials and Transformation }}$}

Marchantia accession Takaragaike-1 (Tak-1, male) and Takaragaike-2 (Tak-2, female) ${ }^{42}$ were used throughout the study. The growth conditions and transformation methods were previously described ${ }^{13,42,43}$. M. polymorpha expressing mCitrine-MpSYP12A, mCitrineMpSYP12B, mCitrine-MpSYP13A, mCitrine-MpSYP13B, mCitrine-MpSYP2 or mCitrineMpVAMP71 under the regulation of their own regulatory elements (e.g. promoter, UTRs, and introns), and $M$. polymorpha expressing mRFP-MpSYP6A or GFP-HDEL driven by the $\mathrm{Mp} E F 1 \alpha$ promoter were previously reported ${ }^{13,38,44}$.

\section{Genotyping}

Genomic DNA was extracted from the thalli in an extraction buffer containing $1 \mathrm{M} \mathrm{KCl}$, $100 \mathrm{mM}$ Tris- $\mathrm{HCl}$ ( $\mathrm{pH} 9.5$ ), and 10 mM EDTA. This DNA was used as templates for PCR. Genome fragments with putative mutation sites were amplified by PCR using KOD FX Neo (TOYOBO, Osaka, Japan) according to the manufacturer's instructions. The primers used in PCR-based genotyping are listed in Supplementary Table S2.

\section{Confocal laser scanning microscopy}

Two- and five-day-old thalli were used for imaging the cell plate and the oil body, respectively. Samples were mounted in distilled water and observed using an LSM780 confocal microscope (Carl Zeiss) equipped with an oil immersion lens $(63 \times$, numerical aperture $=1.4)$ on an electrically driven stage. Gemmae were observed with a $10 \times$ objective lens (numerical aperture $=0.45)$. Thalli were incubated in $10 \mu \mathrm{M} N$-(3-triethylammoniumpropyl)-4-(6-(4(diethylamino)phenyl)hexatrienyl) pyridinium dibromide (FM4-64, Thermo Fisher) solution for 2 min. Samples were then washed twice in water before observation. For 4,4-difluoro-1,3,5,7,8pentamethyl-4-bora-3a,4a-diaza-s-indacene (BODIPY 493/503, Thermo Fisher) staining, thalli or gemmae were incubated in $200 \mathrm{nM}$ BODIPY 493/503 dissolved in water for $10 \mathrm{~min}$ and then washed twice in water before observation. The samples were excited at $488 \mathrm{~nm}$ (Argon 488) and $561 \mathrm{~nm}$ (DPSS 561-10) lasers, respectively, and the fluorescent emission was collected between 482-659 nm using twenty GaAsP detectors. Spectral unmixing and constructing maximum intensity projection images were conducted using the ZEN2012 software (Carl Zeiss). Image 
processing was performed with ZEN2012 software, Photoshop software (Adobe Systems), and ImageJ (National Institute of Health, https://imagej.nih.gov/ij/).

Electron microscopy

Five-day-old M. polymorpha thalli from Tak-1 and thalli expressing mCitrineMpSYP12B were used for the immunoelectron microscopic observation. Sample preparation and observation were performed as previously reported ${ }^{13}$. Five-day-old Tak-1 thalli were used for morphological observation with a transmission electron microscope. Sample preparation and observation were performed as previously reported ${ }^{44}$.

\section{Light sheet microscopy}

Five-day-old thalli expressing $2 \times$ Citrine driven by the $\mathrm{Mp} S Y P 12 B$ promoter were embedded in low melt agarose gel and observed using a Light sheet Z.1 microscope (Carl Zeiss) equipped with a water immersion lens $(5 \times$, numerical aperture $=0.16)$. The samples were excited at 488 $\mathrm{nm}$ (Argon 488 laser). Acquisition and construction of three-dimension images from multi-angle images were conducted using the ZEN2014 software (Carl Zeiss). The images were processed digitally with Imaris (Bitplane) and Photoshop software.

\section{Fluorescent stereoscopic microscopy}

Three-week-old wild-type and Mperf1 $3^{g e}$ thalli were stained by BODIPY493/503 (ThermoFisher) and observed using a fluorescent stereoscopic microscope (M165 FC, Leica) equipped with a MC170 HD digital camera (Leica).

\section{Forward genetic screening for the oil body formation mutants}

The workflow of the mutant screening is described in Fig. S4A. Liverworts with transfer DNA (T-DNA) insertions were generated by co-culture of $M$. polymorpha sporelings with the agrobacteria strain GV2260 harbouring the binary vector pCAMBIA1300 (https://cambia.org/welcome-to-cambialabs/cambialabs-projects/cambialabs-projects-legacypcambia-vectors-pcambia-legacy-vectors-1/cambialabs-projects-legacy-pcambia-vectors-list-oflegacy-pcambia-vectors-3/). $\mathrm{T}_{1}$ plants were first selected on 1/2× Gamborg's B5 plates containing $10 \mathrm{mgL}^{-1}$ hygromycin and $100 \mathrm{mgL}^{-1}$ cefotaxime. Hygromycin-resistant $\mathrm{T}_{1}$ plants 
were then transferred and incubated on hygromycin-free medium for about one week, followed by BODIPY 493/503 staining to visualize oil bodies. Visible screening for mutants defective in oil body formation was performed using a fluorescent stereoscopic microscope (M165 FC, Leica). To identify flanking sequences of T-DNA insertions, thermal asymmetric interlaced-PCR (TAIL-PCR) was performed as described previously ${ }^{42,45,46}$ with minor modifications using crude-extracted DNA as templates. Flanking sequences were amplified using KOD FX neo DNA polymerase and T-DNA-specific (TR1-3 and TL1-3 for right and left borders of T-DNA, respectively) and universal adaptor (AD1-6) primers. The reaction cycles are shown below. After agarose gel electrophoresis of the final TAIL-PCR products, DNA bands were excised and purified using the Wizard SV Gel and PCR Clean-Up System (Promega). Purified products were directly sequenced using TR3 or TL3 primers. The T-DNA insertion sites were identified using the genome sequence registered in MarpolBase, genome version 3.1. Cycling conditions for TAIL-PCR

\begin{tabular}{|c|c|c|}
\hline Reaction & Cycle No. & Thermal settings \\
\hline \multirow[t]{8}{*}{ Primary } & 1 & $94^{\circ} \mathrm{C} 1 \mathrm{~min}, 95^{\circ} \mathrm{C} 1 \mathrm{~min}$ \\
\hline & 5 & $94^{\circ} \mathrm{C} 1 \mathrm{~min}, 65^{\circ} \mathrm{C} 1 \mathrm{~min}, 68^{\circ} \mathrm{C} 3 \mathrm{~min}$ \\
\hline & 1 & $94^{\circ} \mathrm{C} 1 \mathrm{~min}, 30^{\circ} \mathrm{C} 1 \mathrm{~min}$ \\
\hline & & Ramping to $68^{\circ} \mathrm{C} 3 \mathrm{~min}, 68^{\circ} \mathrm{C} 3 \mathrm{~min}$ \\
\hline & 13 & $94^{\circ} \mathrm{C} 30 \mathrm{sec}, 68^{\circ} \mathrm{C} 4 \mathrm{~min}$ \\
\hline & & $94^{\circ} \mathrm{C} 30 \mathrm{sec}, 68^{\circ} \mathrm{C} 4 \mathrm{~min}$ \\
\hline & & $94^{\circ} \mathrm{C} 30 \mathrm{sec}, 44^{\circ} \mathrm{C} 1 \mathrm{~min}, 68^{\circ} \mathrm{C} 3 \mathrm{~min}$ \\
\hline & 1 & $68^{\circ} \mathrm{C} 5 \mathrm{~min}$ \\
\hline \multirow[t]{5}{*}{ Secondary } & 1 & $94^{\circ} \mathrm{C} 1 \mathrm{~min}, 95^{\circ} \mathrm{C} 1 \mathrm{~min}$ \\
\hline & 13 & $94^{\circ} \mathrm{C} 30 \mathrm{sec}, 68^{\circ} \mathrm{C} 4 \mathrm{~min}$ \\
\hline & & $94^{\circ} \mathrm{C} 30 \mathrm{sec}, 68^{\circ} \mathrm{C} 4 \mathrm{~min}$ \\
\hline & & $94^{\circ} \mathrm{C} 30 \mathrm{sec}, 44^{\circ} \mathrm{C} 1 \mathrm{~min}, 68^{\circ} \mathrm{C} 3 \mathrm{~min}$ \\
\hline & 1 & $68^{\circ} \mathrm{C} 5 \mathrm{~min}$ \\
\hline \multirow[t]{5}{*}{ Tertiary } & 1 & $94^{\circ} \mathrm{C} 1 \mathrm{~min}, 95^{\circ} \mathrm{C} 1 \mathrm{~min}$ \\
\hline & 13 & $94^{\circ} \mathrm{C} 30 \mathrm{sec}, 68^{\circ} \mathrm{C} 4 \mathrm{~min}$ \\
\hline & & $94^{\circ} \mathrm{C} 30 \mathrm{sec}, 68^{\circ} \mathrm{C} 4 \mathrm{~min}$ \\
\hline & & $94^{\circ} \mathrm{C} 30 \mathrm{sec}, 44^{\circ} \mathrm{C} 1 \mathrm{~min}, 68^{\circ} \mathrm{C} 3 \mathrm{~min}$ \\
\hline & 1 & $68^{\circ} \mathrm{C} 5 \mathrm{~min}$ \\
\hline
\end{tabular}




\section{RNA extraction and RNA sequencing analysis}

Total RNA from Tak-1, Mperf13 $3^{G O F}$ and Mperf13-1 ${ }^{\text {ge }}$ thalli was extracted by RNeasy (QIAGEN) according to the manufacturer's instructions. The eluted total RNA samples were treated with DNase I (Takara) to remove DNA contamination. The quality and quantity of the total RNA were evaluated with a NanoDrop 1000 spectrophotometer (Thermo Fisher Scientific), Qubit 2.0 Fluorometer (Thermo Fisher Scientific) and a Bioanalyzer RNA6000 Nano Kit (Agilent Technologies). The sequence libraries were prepared with the TruSeq RNA Sample Prep Kit v2 (Illumina) according to the manufacturer's low sample protocol. The quality and quantity of each library were determined using a Bioanalyzer with the High Sensitivity DNA kit (Agilent Technologies) and the KAPA Library Quantification Kit for Illumina (Illumina). Equal amounts of each library were mixed to make the $2 \mathrm{nM}$ pooled library. Illumina sequencing was performed using a HiSeq 1500 platform (Illumina) to produce 126-bp single-end reads. Three biological replicates were prepared for the library construction and RNA-Seq analysis. All reads are available through the Sequence Read Archive (SRA) under the accession number DRA009193. The abundance of transcripts from Illumina RNA-Seq data were quantified with the kallisto program (version 0.43 .1 ) ${ }^{47}$ with the default parameters using the primary transcript dataset from the Marchantia genome version 3.1. To estimate differentially expressed genes (DEGs), an ANOVA-like test was performed by edgeR (version 3.18.1) ${ }^{48}$. Genes with FDR values lower than 0.01 and an absolute $\log _{2}$ fold change $(\mathrm{FC})>2$ were considered to be differentially expressed. Among all combinations of DEGs $\left(\log _{2} \mathrm{FC}\left(\mathrm{Mperf13}{ }^{G O F} / \mathrm{Tak}-1\right)>2\right.$ and $\log _{2} \mathrm{FC}\left(\right.$ Mperf13-1 ${ }^{\text {ge }} /$ Tak-1) $>2$, $\log _{2} \mathrm{FC}\left(\right.$ Mperf13 $3^{\text {GOF}} /$ Tak-1 $)>2$ and $\log _{2} \mathrm{FC}\left(\right.$ Mperf13-1 ${ }^{\text {ge }} /$ Tak-1) $<-2, \log _{2} \mathrm{FC}\left(\right.$ Mperf13 $3^{G O F} /$ Tak-1 $)<-2$ and $\log _{2} \mathrm{FC}\left(\right.$ Mperf13-1 ${ }^{\text {ge }} /$ Tak-1 $)>2$, $\log _{2} \mathrm{FC}\left(\right.$ Mperf13 $3^{G O F} /$ Tak-1 $)<-2$ and $\log _{2} \mathrm{FC}\left(\right.$ Mperf13-1 ${ }^{g e} /$ Tak-1 $\left.)<-2\right)$, we selected the set $\log _{2} \mathrm{FC}\left(\right.$ Mperf13 ${ }^{G O F} /$ Tak-1) $>2$ and $\log _{2} \mathrm{FC}\left(\right.$ Mperf13-1 $1^{g e} /$ Tak-1 $)<-2$ as candidate genes regulated downstream of MpERF13, which included both MpERF13 and MpSYP12B.

\section{Quantitative reverse transcription (qRT)-PCR}

For qRT-PCR, cDNA was synthesized from total RNA using SuperScript III Reverse Transcriptase (Invitrogen) and an oligo dT(18) primer according to the manufacturer's instructions. qRT-PCR was performed with LightCycler 480 (Roche) using FastStart SYBR Green Master (Roche) according to the manufacturer's protocol. Sequences of primers used are 
listed in Table S2. MpAPT (Mapoly0100s0027.1 / Mp3g25140.1) was used as a housekeeping reference for normalization ${ }^{49}$. Three biological replicates were prepared and three technical replicates were performed for each reaction.

\section{Pill bug feeding assay}

Feeding assay was performed according to Nakazaki et al. with modification ${ }^{50}$. Gemmae were cultured on 1/2× Gamborg's B5 medium containing 1\% (w/v) agar and 1\% (w/v) sucrose for five days at $22^{\circ} \mathrm{C}$ under continuous white light $\left(50 \mu \mathrm{mol} \mathrm{m} \mathrm{m}^{-2}\right)$. Five-day-old thalli were transferred onto 1/2× Gamborg's B5 medium containing 1\% (w/v) agar without sucrose and cultured for additional five days under the same conditions. Pill bugs (Armadillidium vulgare) were collected in the Myodaiji area of NIBB (Nishigonaka 38, Myodaiji, Okazaki 444-8585 Aichi, Japan). Pill bugs were maintained on Prowipe (Daio Paper, Tokyo, Japan) moistened with sterilized water for $48 \mathrm{~h}$ at $22^{\circ} \mathrm{C}$ in the dark without feeding before the assay. Six pill bugs were introduced into each medium plate containing 10-day-old thalli and kept for $24 \mathrm{~h}$ in the dark at $22^{\circ} \mathrm{C}$. The thallus areas were calculated using ImageJ (National Institute of Health, https://imagej.nih.gov/ij/).

Gene accession numbers

\begin{tabular}{lll}
\hline Symbol & Gene ID (ver3.1) & Gene ID (ver5.1) \\
\hline MpSYP12A & Mapoly0163s0015 & Mp6g00050 \\
\hline MpSYP12B & Mapoly0101s0013 & Mp4g20670 \\
\hline MpSYP13A & Mapoly0009s0005 & Mp7g13190 \\
\hline MpSYP13B & Mapoly0055s0091 & Mp2g19600 \\
\hline MpCLC1 & Mapoly0075s0069 & Mp2g03080 \\
\hline MpPIP2 & Mapoly0041s0003 & Mp4g17210 \\
\hline MpERF13 & Mapoly0060s0052 & Mp6g08690 \\
\hline MpABCG1 & Mapoly0083s0014 & Mp8g13070 \\
\hline MpMYB02 & Mapoly0006s0226 & Mp3g07510 \\
\hline MpTUB2 & Mapoly0158s0010 51,52 & Mp2g09390 \\
\hline MpSYP6A & Mapoly0140s0004 & Mp3g18380 \\
\hline MpSYP2 & Mapoly0187s0013 & Mp8g15260 \\
\hline MpSFT1 & Mapoly0006s0109 & Mp3g06390 \\
\hline MpGOS11 & Mapoly0001s0245 & Mp1g19070 \\
\hline MpSYP4 & Mapoly0042s0041 & Mp2g14140 \\
\hline Mp VAMP71 & Mapoly0064s0109 & Mp8g00880 \\
\hline MpSEC20 & Mapoly0019s0137 & Mp1g13670
\end{tabular}


641 We followed the nomenclature of genes, proteins, and mutants of Marchantia reported in

642 Bowman et al. (2016) ${ }^{53}$. Gene IDs were taken from MarpolBase (http://marchantia.info/), 643 genome version 3.1 and version $5.1^{20,54}$.

644 

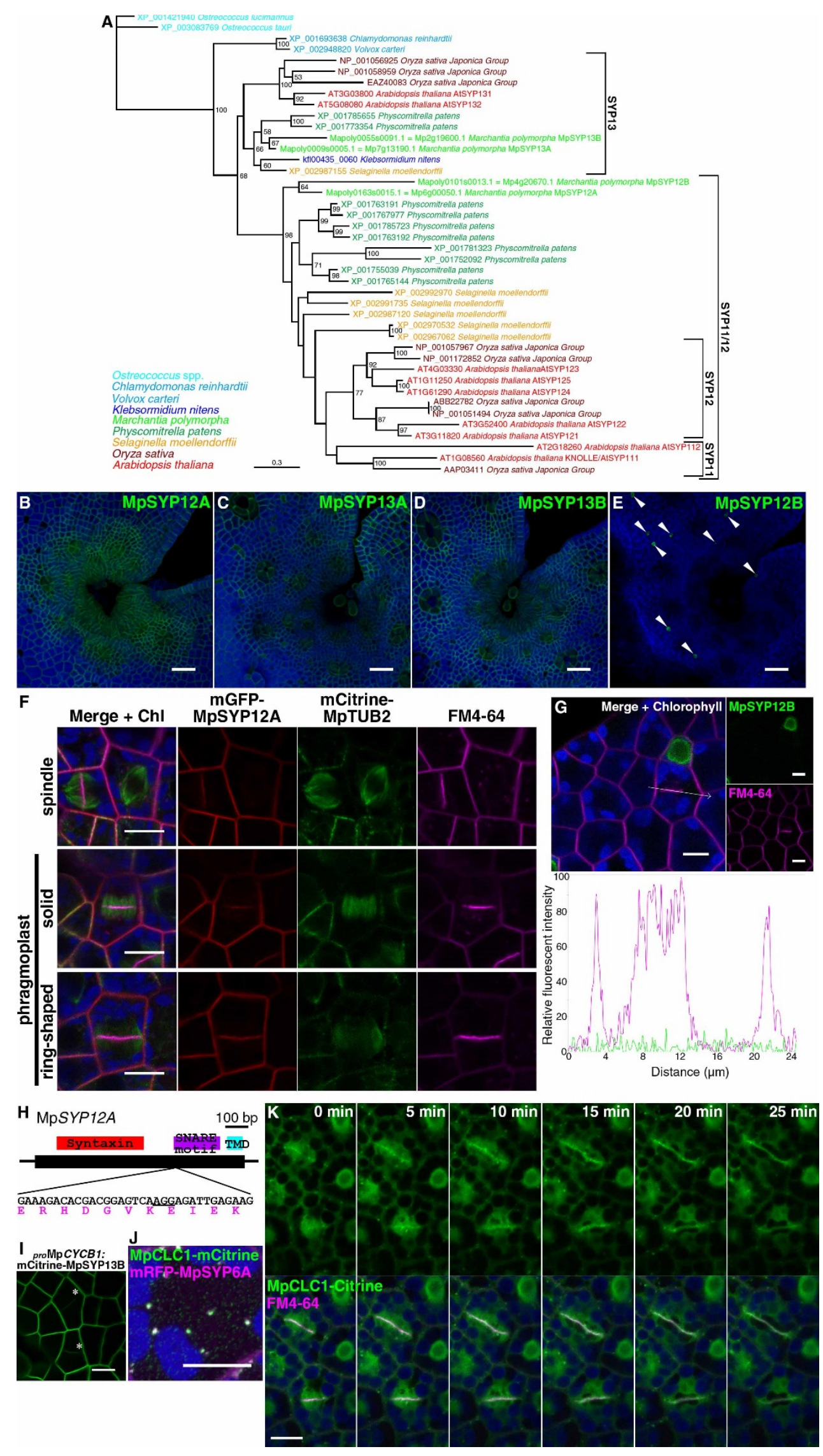


\section{cytokinesis in Marchantia thalli}

(A) A maximum likelihood phylogenetic tree of SYP1 members in green plants. Although the tree is unrooted, the proteins from Chlorophytes (Ostreococcus spp., Chlamydomonas reinhardtii, and Volvox carteri) are the outgroup to those of streptophytes. The branch lengths are proportional to the estimated number of substitutions per site. Bootstrap probability is indicated as a percentage on each branch with at least 50\% support. (B - E) Maximum intensity projection images of thalli expressing mCitrine-MpSYP12A (B), mCitrine-MpSYP13A (C), mCitrine-MpSYP13B (D), or mCitrine-MpSYP12B (E). White arrowheads indicate mCitrineMpSYP12B-expressing cells. (F) Single confocal images of thallus cells expressing mCitrineMpTUB2 and mGFP-MpSYP12A under the regulation of the MpSYP2 and MpSYP12A promoters, respectively, stained with FM-4-64. In mitotic cells, spindles and phragmoplasts (solid and ring-shaped phases) were labelled by mCitrine-MpTUB2, and endocytosed FM-4-64 accumulated at forming cell plates. (G) Single confocal images of thallus cells expressing mCitrine-MpSYP12B stained with FM4-64. The line graph indicates the relative fluorescence intensity along the white arrow. Relative fluorescent intensity of mCitrine to fluorescence on the oil body membrane is shown for mCitrine fluorescence. $(\mathrm{H})$ The gene model and sequences of MpSYP12A. The syntaxin domain, SNARE motif, and transmembrane domain (TMD) are shown above the gene model. Black and magenta letters indicate genome and translated amino acid sequences. The protospacer adjacent motif (PAM) sequence for gRNA is underlined. (I) Expression by the $\mathrm{MpCYCB} 1$ promoter results in accumulation of MpSYP13B at the forming cell plate. A single confocal image of dividing thallus cells expressing mCitrine-MpSYP13B are shown. Asterisks indicate forming cell plates. (J) A maximum intensity projection image of a thallus cell expressing MpCLC1-mCitrine and mRFP-MpSYP6A under the regulation of their own regulatory elements and the $\mathrm{MpEF1 \alpha}$ promoter, respectively. (K) Time-lapse single confocal images of thallus cells expressing MpCLC1-Citrine under the regulation of the MpEF $1 \alpha$ promoter (upper panels) and merged images with FM4-64 signals. Autofluorescence from chlorophyll is pseudo-colored in blue. Bars $=50 \mu \mathrm{m}$ in (B - E) and $10 \mu \mathrm{m}$ in (F), (G), (I), $(\mathrm{J})$, and $(\mathrm{K})$. 


\section{A}

PMpGWB301

proMpSYP12B:mCitrine-GW

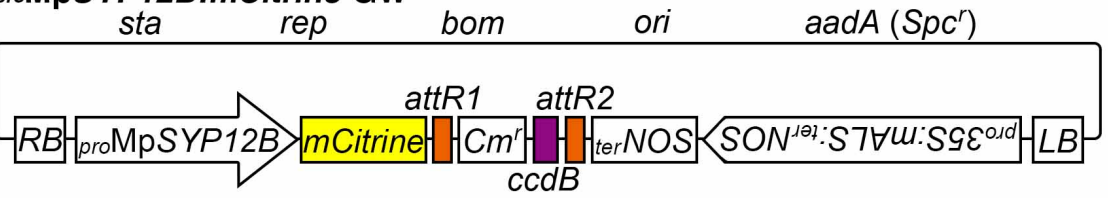

pMpGWB301

proMpSYP2:mCitrine-GW

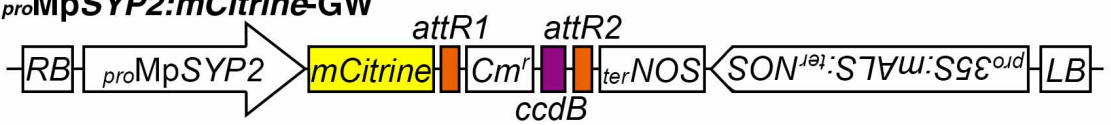

PMPGWB301

proMpSYP12A:mCitrine-GW attR1 attR2

pMpGWB301

proMpCYCB1:mCitrine-GW

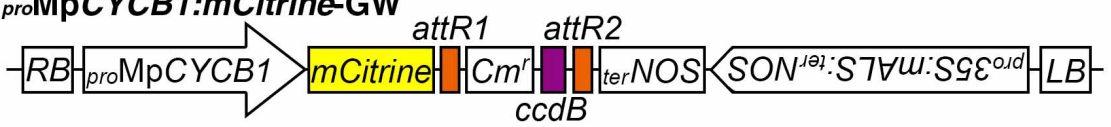

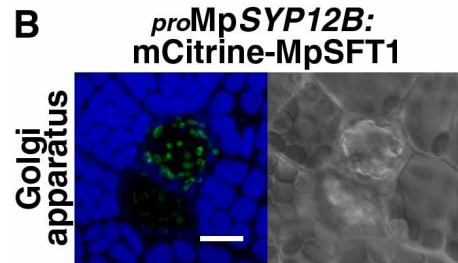

proMpSYP12B:

mCitrine-MpSYP4

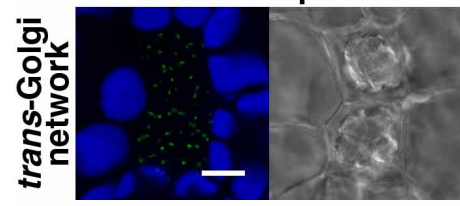

proMpSYP12B:

mCitrine-MpSYP2

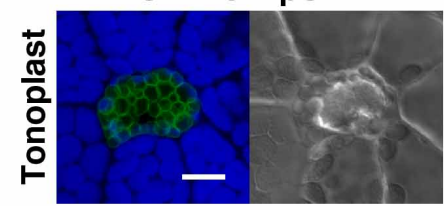

C
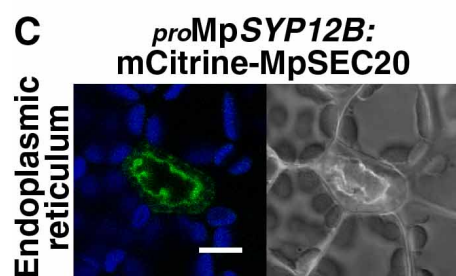

proMpSYP2: mCitrine-MpSFT1

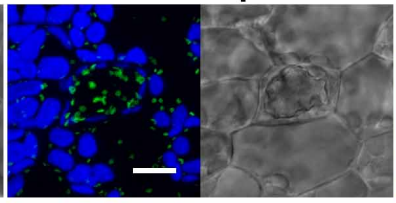

proMpSYP4: mCitrine-MpSYP4

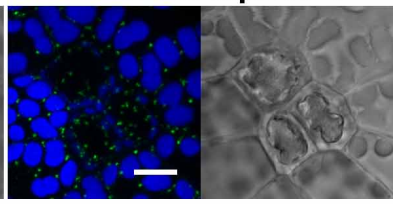

proMpSYP2: mCitrine-MpSYP2

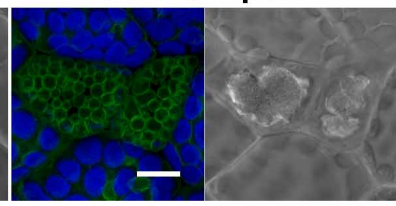

proMpSYP12B:

mCitrine-MpUSE1A

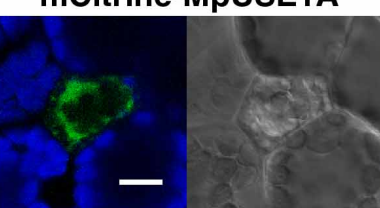

proMpSYP12B: mCitrine-MpGOS11

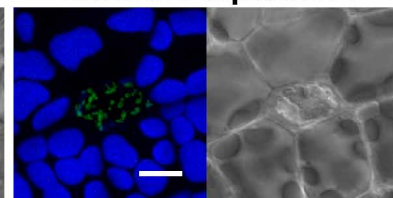

proMpSYP12B:

mCitrine-MpSYP6A

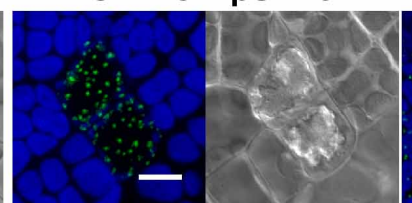

proMpSYP12B: mCitrine-MpVAMP71

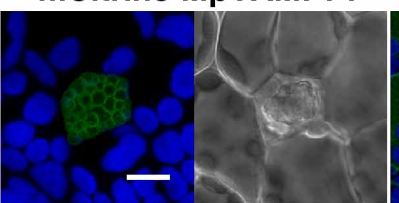

proMpSEC22:

mCitrine-MpSEC22

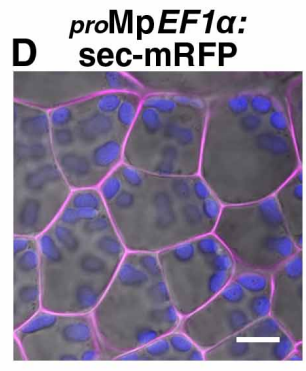

proMpSYP2:

mCitrine-MpGOS11

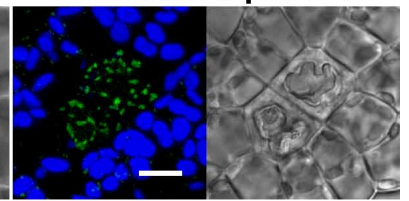

proMpSYP2:

mCitrine-MpSYP6A

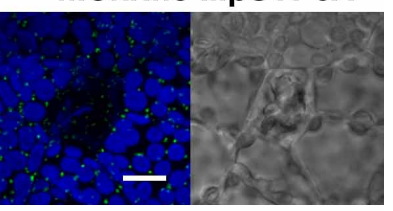

proMp VAMP71: mCitrine-MpVAMP71

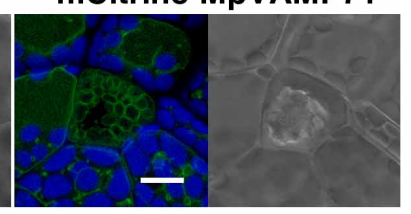

proMpEF1a:

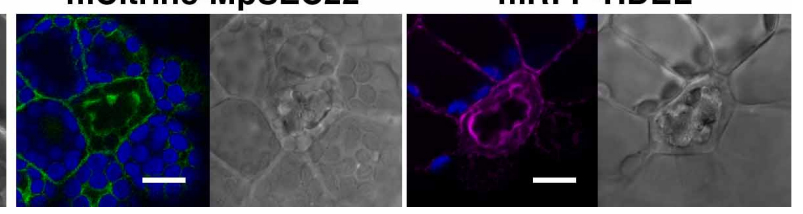

Fig. S2. Subcellular localization of organelle markers in oil body cells

(A) Gateway constructs used in this study. pMpGWB301 proMpSYP2:mCitrine-GW was

previously prepared $^{44}$. (B) Maximum intensity projection images of thallus cells including the oil 
body cell expressing mCitrine-fused markers for the Golgi apparatus (MpSFT1 and MpGOS11), trans-Golgi network (MpSYP4 and MpSYP6A), and tonoplast (MpSYP2 and MpVAMP71). The organelle markers were expressed under the MpSYP12B, MpSYP2, and/or own promoters. Bright field images are also shown. (C) Single confocal images of thallus cells including the oil body cell expressing endoplasmic reticulum markers. mCitrine-MpSEC20 and mCitrineMpUSE1A were expressed under the MpSYP12B promoter. mCitrine-MpSEC22 and mRFPHDEL were expressed using their own regulatory elements and the MpEF1 $\alpha$ promoter, respectively. (D) A single confocal image of thallus cells expressing sec-mRFP expressed under the $\mathrm{Mp} E F 1 \alpha$ promoter. Green, magenta, and blue pseudo-colors indicate fluorescence from mCitrine, mRFP, and chlorophyll, respectively. Bars $=10 \mu \mathrm{m}$. 


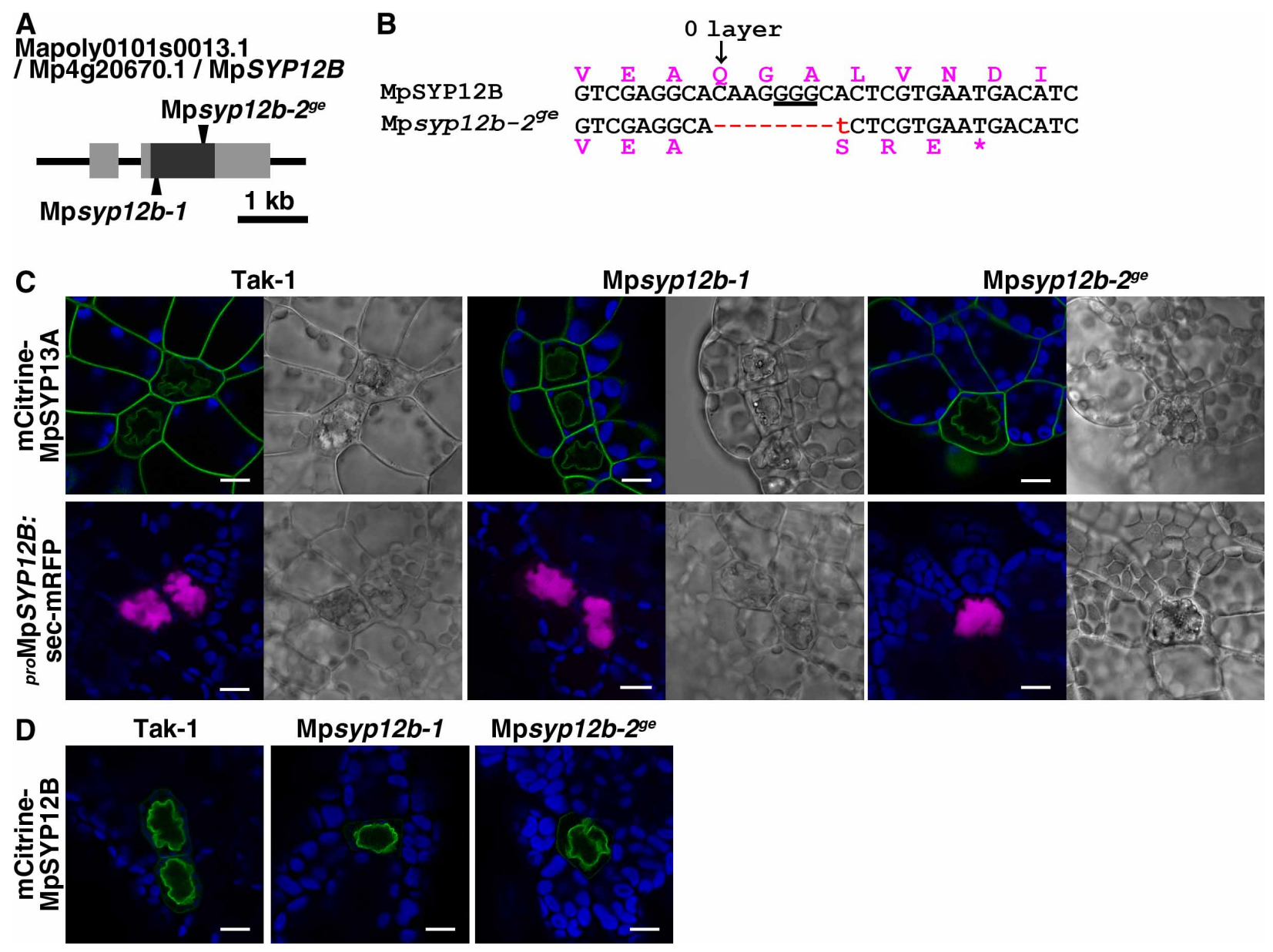

Fig. S3. Phenotype of Mpsyp12b mutants

(A) Schematic representation of the MpSYP12B gene structure. Gray and black boxes indicate untranslated and coding regions, respectively, and arrowheads indicate mutation sites in two independently generated mutants. The Mpsyp 12b-1 mutant was generated by a homologous recombination-mediated gene targeting method $^{41}$. (B) The genome (black letters) and translated amino acid (magenta letters) sequences of $M p S Y P 12 B$ and the Mpsyp 12b-2 ${ }^{\text {ge }}$ mutant, with the red letters indicating the mutated region. The PAM sequence for gRNA is underlined. The 0 layer at the centre of the SNARE motif is shown. (C) Single confocal images of thallus cells including the oil body cell expressing mCitrine-MpSYP13A under its own regulatory elements, or sec-mRFP under the MpSYP12B promoter in Tak-1, Mpsyp12b-1, and Mpsyp12b-2 $2^{g e}$ lines. Bright field images are also shown. (D) Single confocal images of thallus cells including the oil body cell expressing mCitrine-MpSYP12B under its own regulatory elements in Tak-1 and Mpsyp $12 b$ mutants. Green, magenta, and blue pseudo-colors indicate fluorescence from mCitrine, mRFP, and chlorophyll, respectively. Bars $=10 \mu \mathrm{m}$. 
A

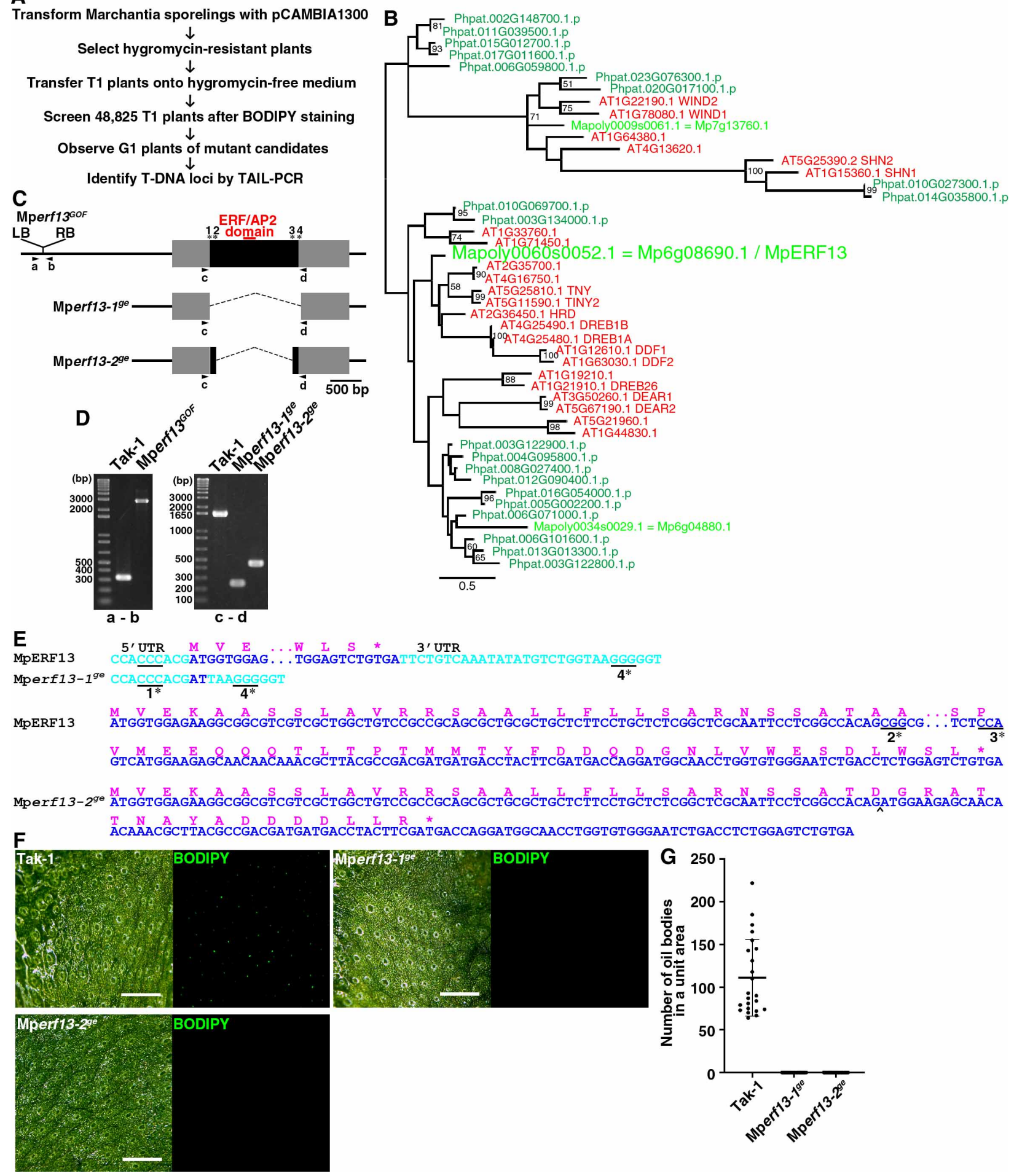

Fig. S4. Screening and generation of Mperf13 mutants

(A) The flow chart of the screen for mutants defective in oil body formation from T-DNAinsertion lines. (B) A maximum likelihood phylogenetic tree of proteins with one ERF/AP2 domain. The color code is shown in Fig S1A. The branch lengths are proportional to the 
estimated number of substitutions per site. Bootstrap probability is indicated as a percentage on each branch with at least 50\% support. A more detailed tree was presented previously (20). (C) Schematic representation of the $\mathrm{MpERF13}$ gene structure and mutations generated in this study. Gray and black boxes indicate the UTR and coding sequences, respectively. Asterisks with numbers indicate sites of designed gRNA to generate Mperf13-19e (*1 and *4) and Mperf13-2 ${ }^{\text {ge }}$ (*2 and *3). (D) PCR-based genotyping of Tak-1, Mperf13 ${ }^{G O F}$, Mperf13-1 ${ }^{g e}$, and Mperf13-2 ${ }^{\text {ge }}$. The combinations and annealing sites of primers (a to d) are shown in (C). (E) The genomic and predicted amino acid sequences of the MpERF13 locus in Tak-1 and Mperf13 mutants. Light blue, dark blue, and magenta letters indicate UTR, coding, and predicted amino acid sequences, respectively. The PAM sequences for gRNAs are underlined. The caret indicates the indel site. (F) Fluorescent and bright-field images of BODIPY-stained three-week-old thalli of Tak-1, Mperf13-1 $1^{g e}$, and Mperf13-2ge. Bars $=0.5 \mathrm{~mm}$. (G) The number of oil bodies visualized with BODIPY in a unit area $(2.0 \mathrm{~mm} \times 2.0 \mathrm{~mm})$. Bars indicate means $\pm \mathrm{SD}$. Statistical analyses between Tak-1 and each genotype were conducted using a two-tailed Welch's $t$-test. Sample numbers were 23 thalli for Tak-1, 24 for Mperf13-1 ${ }^{\text {ge }}$, and 26 for Mperf13-2ge. $p$-values are $5.14 \times 10^{-11}$ for Mperf $13-1^{g e}$ and $5.14 \times 10^{-11}$ for Mperf $13-2^{g e}$. 
A

\begin{tabular}{|c|c|c|c|c|}
\hline Ver3.1 Gene & ID ${ }_{\text {Ver5.1 }}$ & Description & $\begin{array}{c}\log F C \\
\text { (Mperf13 }^{\text {GOF }} / \text { Tak-1) }\end{array}$ & $\begin{array}{c}\text { logFC } \\
\text { (Mperf13-19e/Tak-1) }\end{array}$ \\
\hline Mapoly0083s0014 & Mp8g13070 & $A B C$ transporter & 4.273 & -3.722 \\
\hline Mapoly2351s0001 & Mp2g20510 & Cytochrome P450 & 6.795 & -5.717 \\
\hline Mapoly0119s0008 & Mp4g14860 & LEA protein & 5.045 & -3.099 \\
\hline Mapoly0101s0013 & Mp4g20670 & MpSYP12B & 4.374 & -5.777 \\
\hline Mapoly0006s0226 & Mp3g07510 & MpMYB02 & 3.053 & -7.208 \\
\hline Mapoly0060s0052 & Mp6g08690 & MpERF13 & 4.734 & -5.205 \\
\hline Mapoly0110s0019 & Mp8g13380 & Cellulase & 4.005 & -6.537 \\
\hline Mapoly0005s0135 & Mp1g04730 & Lipid transfer & 4.454 & -4.903 \\
\hline Mapoly0081s0021 & Mp8g05200 & Receptor-like kinase & 2.813 & -4.406 \\
\hline Mapoly0060s0009 & Mp6g09100 & Ankyrin repeats & 4.243 & -5.086 \\
\hline Mapoly0207s0009 & Mp2g24710 & Tomosyn12 & 3.698 & -5.704 \\
\hline
\end{tabular}

B Mapoly0083s0014 Mapoly2351s0001 Mapoly0119s0008 $\quad$ Mapoly0101s0013
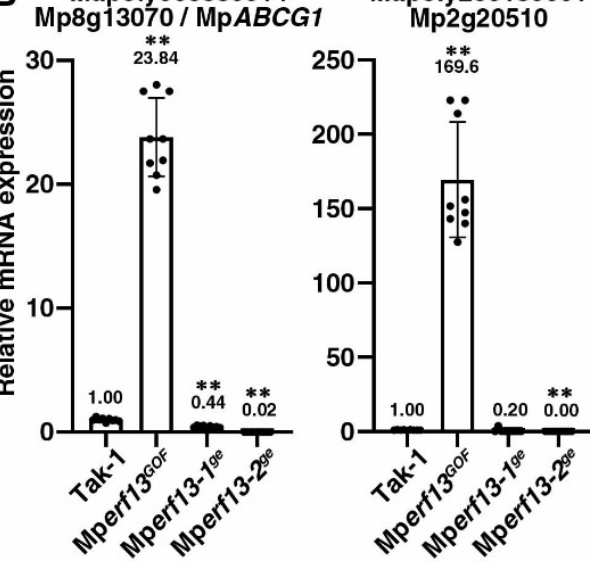
Mp4g14860
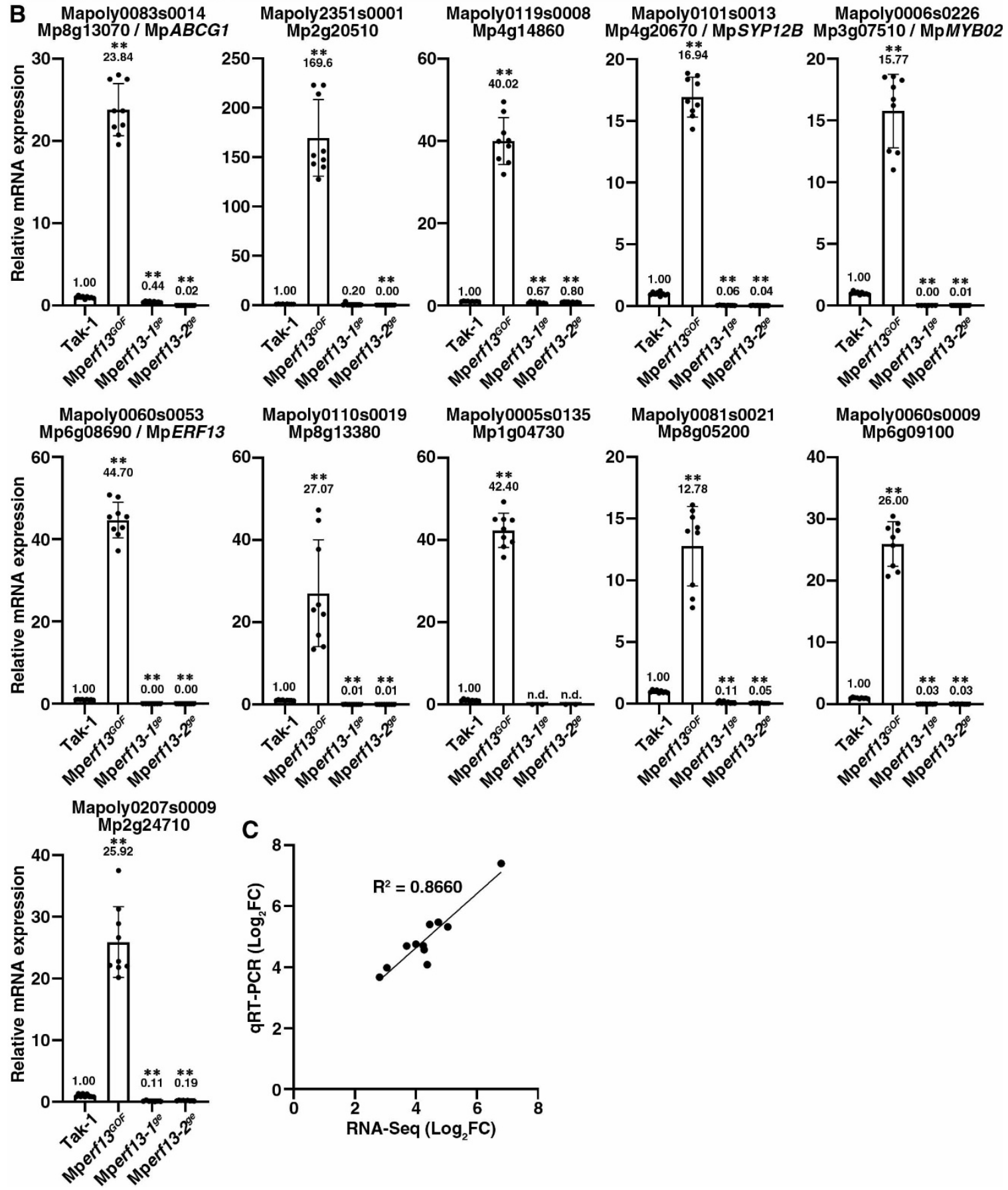
(A) List of selected differentially expressed genes (DEGs) for qRT-PCR analysis. The full list of DEGs are shown as Supplementary Table S1. (B) The relative mRNA expression of 11 DEGs in Tak-1, Mperf13 ${ }^{G O F}$, Mperf13-1 ${ }^{g e}$, and Mperf13-2 $2^{g e}$ measured by qRT-PCR. MpAPT was used as an internal reference. Error bars represent \pm SD. Means are indicated above the bars. Statistical comparison between Tak-1 and each genotype was conducted with a two-tailed Welch's $t$-test. **, $p<0.01$. (C) Correlation of the expression levels between the RNA-Seq analysis and qRTPCR experiment. Both the $\mathrm{x}$ - and $\mathrm{y}$ - axes are shown in a $\log _{2} \mathrm{FC}$ scale. $\mathrm{R}^{2}$ indicates the correlation coefficient. 

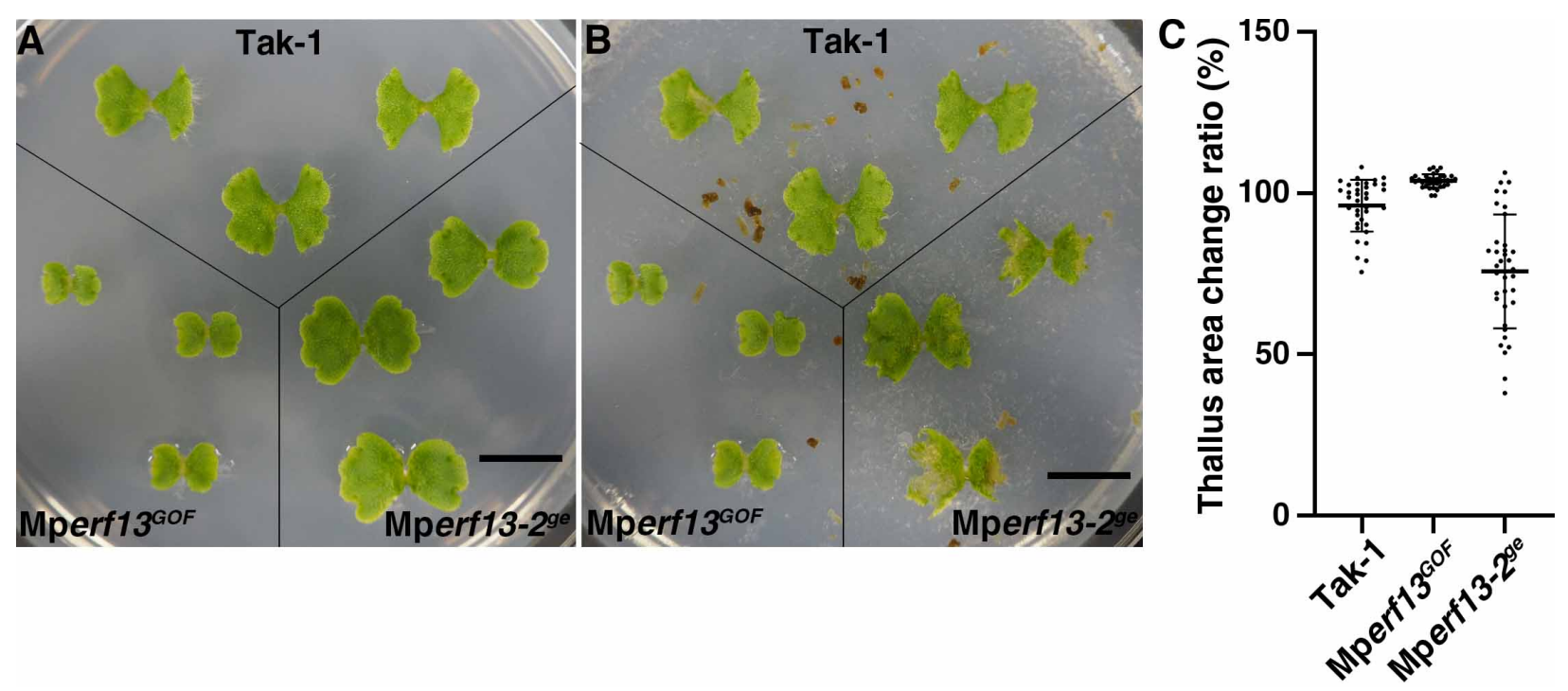

(A and B) Ten-day-old thalli of Tak-1, Mperf13 ${ }^{G O F}$ and Mperf13-2 ${ }^{g e}$ (A) were co-cultivated with starved pill bugs for $24 \mathrm{hr}(\mathrm{B})$. Bars $=1 \mathrm{~cm}$. (C) Ratios of thallus area before and after cocultivation with pill bugs were calculated $(n=36$ thalli for each genotype). Bars indicate means \pm SD. Statistical analyses between Tak-1 and each genotype were conducted with a two-tailed 
Table S1. The list of DEGs in Mperf13 mutants identified in RNA-Seq analysis.

Table S2. List of primers used in this study.

\section{Movie S1. Formation of clathrin-coated vesicles at the oil body membrane.}

A time-lapse movie of an oil body cell expressing MpCLC1-Citrine. Green and blue pseudocolors represent fluorescence from Citrine and chlorophyll, respectively. $32 \times$ real time. Bar $=5$ $\mu \mathrm{m}$.

\section{Movie S2. Expression of MpSYP12B in a Marchantia thallus}

A five-day-old thallus expressing $2 \times$ YFP driven by the MpSYP12B promoter observed by light sheet microscopy. Green and blue pseudo-colors represent fluorescence from Citrine and chlorophyll, respectively. Bar $=400 \mu \mathrm{m}$.

\section{References:}

33 Edgar, R. C. MUSCLE: a multiple sequence alignment method with reduced time and space complexity. BMC bioinformatics 5, 113, doi:10.1186/1471-2105-5-113 (2004).

34 Edgar, R. C. MUSCLE: multiple sequence alignment with high accuracy and high throughput. Nucleic acids research 32, 1792-1797, doi:10.1093/nar/gkh340 (2004).

35 Castresana, J. Selection of conserved blocks from multiple alignments for their use in phylogenetic analysis. Mol Biol Evol 17, 540-552, doi:DOI 10.1093/oxfordjournals.molbev.a026334 (2000).

36 Talavera, G. \& Castresana, J. Improvement of phylogenies after removing divergent and ambiguously aligned blocks from protein sequence alignments. Syst Biol 56, 564-577, doi:10.1080/10635150701472164 (2007). 
37 Guindon, S. et al. New Algorithms and Methods to Estimate Maximum-Likelihood Phylogenies: Assessing the Performance of PhyML 3.0. Syst Biol 59, 307-321, doi:10.1093/sysbio/syq010 (2010).

38 Ishizaki, K. et al. Development of Gateway Binary Vector Series with Four Different Selection Markers for the Liverwort Marchantia polymorpha. PloS one 10, e0138876, doi:10.1371/journal.pone.0138876(2015).

39 Naito, Y., Hino, K., Bono, H. \& Ui-Tei, K. CRISPRdirect: software for designing CRISPR/Cas guide RNA with reduced off-target sites. Bioinformatics 31, 1120-1123, doi:10.1093/bioinformatics/btu743 (2015).

40 Sugano, S. S. et al. Efficient CRISPR/Cas9-based genome editing and its application to conditional genetic analysis in Marchantia polymorpha. PloS one 13, e0205117, doi:10.1371/journal.pone.0205117 (2018).

41 Ishizaki, K., Johzuka-Hisatomi, Y., Ishida, S., Iida, S. \& Kohchi, T. Homologous recombination-mediated gene targeting in the liverwort Marchantia polymorpha L. Scientific reports 3, 1532, doi:10.1038/srep01532 (2013).

42 Ishizaki, K., Chiyoda, S., Yamato, K. T. \& Kohchi, T. Agrobacterium-mediated transformation of the haploid liverwort Marchantia polymorpha L., an emerging model for plant biology. Plant \& cell physiology 49, 1084-1091, doi:10.1093/pcp/pcn085 (2008).

43 Kubota, A., Ishizaki, K., Hosaka, M. \& Kohchi, T. Efficient Agrobacterium-mediated transformation of the liverwort Marchantia polymorpha using regenerating thalli. Bioscience, biotechnology, and biochemistry 77, 167-172, doi:10.1271/bbb.120700 (2013). 
44 Minamino, N. et al. Dynamic reorganization of the endomembrane system during spermatogenesis in Marchantia polymorpha. Journal of plant research 130, 433-441, doi:10.1007/s10265-017-0909-5 (2017).

45 Honkanen, S. et al. The Mechanism Forming the Cell Surface of Tip-Growing Rooting Cells Is Conserved among Land Plants. Curr Biol 26, 3238-3244, doi:10.1016/j.cub.2016.09.062 (2016).

46 Liu, Y. G., Mitsukawa, N., Oosumi, T. \& Whittier, R. F. Efficient Isolation and Mapping of Arabidopsis-thaliana T-DNA Insert Junctions by Thermal Asymmetric Interlaced Pcr. Plant Journal 8, 457-463, doi:DOI 10.1046/j.1365-313X.1995.08030457.x (1995).

47 Bray, N. L., Pimentel, H., Melsted, P. \& Pachter, L. Near-optimal probabilistic RNA-seq quantification. Nature biotechnology 34, 525-527, doi:10.1038/nbt.3519 (2016).

48 Robinson, M. D., McCarthy, D. J. \& Smyth, G. K. edgeR: a Bioconductor package for differential expression analysis of digital gene expression data. Bioinformatics 26, 139140, doi:10.1093/bioinformatics/btp616 (2010).

49 Saint-Marcoux, D., Proust, H., Dolan, L. \& Langdale, J. A. Identification of reference genes for real-time quantitative PCR experiments in the liverwort Marchantia polymorpha. PloS one 10, e0118678, doi:10.1371/journal.pone.0118678 (2015).

50 Nakazaki, A. et al. Leaf Endoplasmic Reticulum Bodies Identified in Arabidopsis Rosette Leaves Are Involved in Defense against Herbivory. Plant physiology 179, 1515-1524, doi:10.1104/pp.18.00984 (2019).

51 Buschmann, H., Holtmannspotter, M., Borchers, A., O'Donoghue, M. T. \& Zachgo, S. Microtubule dynamics of the centrosome-like polar organizers from the basal land plant 

(2016).

52 Otani, K. et al. An evolutionarily conserved NIMA-related kinase directs rhizoid tip growth in the basal land plant Marchantia polymorpha. Development 145, doi:10.1242/dev.154617 (2018).

53 Bowman, J. L. et al. The Naming of Names: Guidelines for Gene Nomenclature in Marchantia. Plant \& cell physiology 57, 257-261, doi:10.1093/pcp/pcv193 (2016). Ancestral Association between H3K27me3, Transposons, and Constitutive Heterochromatin. Curr Biol, doi:10.1016/j.cub.2019.12.015 (2020). 